Supporting Information

\title{
Self-Assembled Open Porous Nanoparticle Superstructures
}

Fenghua Zhang ${ }^{\dagger}$ Rongjuan Liu ${ }^{\dagger}$ Yanze Wei

$\dagger$ Key Laboratory of Colloid and Interface Chemistry, Ministry of Education, School of Chemistry and

Chemical Engineering, Shandong University, Jinan 250100, P.R. China.

*E-mail: zyangchem@sdu.edu.cn 


\section{Table of Contents}

1. Experimental section

2. Molecular dynamics simulations

3. Supplementary note 1

4. Supplementary note 2

5. Supplementary note 3

6. Supplementary note 4

7. Supporting figures

8. Movie captions 


\section{Experimental section}

Materials. All chemicals were used as received without any further purification: $\mathrm{CoSO}_{4} \cdot 7 \mathrm{H}_{2} \mathrm{O}$ (Sinopharm Chemical Reagent Co., Ltd, 99.5\%), $\mathrm{Fe}_{2} \mathrm{O}_{3}$ (Sinopharm Chemical Reagent Co., Ltd, 99.5\%), trimethyltetradecylammonium chloride (TTAC, Sinopharm Chemical Reagent Co., Ltd, 99.5\%), octylamine (Adamas, 99\% ), toluene (Fuyu, 99.5\%), acetone (Fuyu, 99.5\%), sodium oleate (TCI, 97\%), chloroform (Adamas, 99.8\%), 1-octadecene (ODE, TCI, 90\%), ethanol ( $\geq 99 \%$ ), methyl alcohol ( $\geq 99 \%)$, hexane (Sigma-Aldrich, 95\%), oleic acid (OA, Sigma-Aldrich, 70\%), oleylamine (OAM, Sigma-Aldrich, 70\%), chloro(triphenylphosphine)gold (Sigma-Aldrich, 99\%), 1dodecanethiol (DDT, Sigma-Aldrich, 99\%), lithium hydroxide (Sigma-Aldrich, 99\%), gadolinium acetate (Sigma-Aldrich, 99\%), diphenylsilane (Sigma-Aldrich, 98\%), dialkyl phenylsilane (SigmaAldrich, 97\%), n-butyl alcohol (n-BuOH, Adamas, 99.5\%), (-)-limonene (TCI, >99\%), triethylsilane (Macklin, 99\%), N, N-diisopropylethylamine (DIPEA, Macklin, 99\%), phenylacetylene (Macklin, 98\%), dimethyl acetylene dicarboxylate (Macklin, $\geq 98 \%$ ), diethylacetylene (Macklin, $\geq 98 \%$ ), $\mathrm{HAuCl}_{4}$ (Macklin, $\geq 99 \%$ ), Pt(acac) 2 (Macklin, $\geq 97 \%$ ), Pd(acac)2 (Macklin, $\geq 97 \%$ ), trioctylphosphine (TOP, Macklin, $\geq 97 \%$ ), trioctylphosphine oxide (TOPO, Macklin, $\geq 98 \%$ ), n-tetradecylphosphonicacid (TDPA, Sigma-Aldrich, $\geq 98 \%$ ), tributyl phosphate (TBP, Sigma-Aldrich, $\geq 99 \%$ ), 1-phenyl-1-hexyne (Sigma-Aldrich, 98\%).

Synthesis of Au NPs. ${ }^{1}$ In a typical synthesis of Au nanocrystals capped with 1-dodecanethiol, a precursor solution of toluene $(25 \mathrm{~mL})$ and chloro (triphenylphosphine) gold $(0.124 \mathrm{~g})$ was prepared in a three-neck flask $(100 \mathrm{~mL})$ and heated to $100{ }^{\circ} \mathrm{C}$ magnetically stirred under $\mathrm{N}_{2}$ flow for $10 \mathrm{~min}$. A reducing solution containing $0.434 \mathrm{~g}$ of TBAB, toluene $(3 \mathrm{~mL})$ and 1-dodecanethiol $(2 \mathrm{mM})$ was mixed at $70{ }^{\circ} \mathrm{C}$ by sonication and injected into the precursor solution. The reduction was instantaneously initiated and the solution changed to a deep purple color within $5 \mathrm{~s}$. The mixture was allowed to react at $100{ }^{\circ} \mathrm{C}$ for $5 \mathrm{~min}$ before acetone $(60 \mathrm{~mL})$ was added to precipitate the Au nanocrystals. The Au3 nanocrystals was collected by centrifugation $(8000 \mathrm{rpm}, 3 \mathrm{~min})$, washed with acetone and redispersed in hexane. Au NPs with different size were prepared by changing the amount of TBAB and DDT. The diverse amount of TBAB and DDT as described in Table S1. In addition, Au13 NPs was synthesized by the method of seed growth: a seed solution of toluene $(2 \mathrm{~mL})$ and Au5 $(0.5 \mathrm{mg})$ was prepared in a $20-\mathrm{mL}$ glass. A solution containing $15 \mathrm{mg}$ of $\mathrm{HAuCl}_{4}$, toluene $(11 \mathrm{~mL})$ and $\mathrm{OAM}(2 \mathrm{~mL})$ was mixed at $90{ }^{\circ} \mathrm{C}$ for 5 hours. Then, ethanol $(20 \mathrm{~mL})$ was added to precipitate the Au13 NPs. The Au13 NPs was collected by centrifugation ( $8000 \mathrm{rpm}, 3 \mathrm{~min})$ and redispersed in hexane. 
Table S1. Au NPs with different size were prepared by changing the amount of TBAB and DDT.

\begin{tabular}{cccc}
\hline Entry & Chloro(triphenylphosphine)gold/mg & TBAB/mg & DDT/ $\boldsymbol{\mu L}$ \\
\hline Au3 $(3.1 \mathrm{~nm})$ & 124 & 450 & 500 \\
Au4 $(4.5 \mathrm{~nm})$ & 124 & 300 & 500 \\
Au5 $(5.1 \mathrm{~nm})$ & 124 & 200 & 500 \\
Au6 $(6.1 \mathrm{~nm})$ & 124 & 200 & 250 \\
Au7 $(7.1 \mathrm{~nm})$ & 124 & 100 & 150 \\
\hline
\end{tabular}

Synthesis of $\mathrm{Fe}_{3} \mathrm{O}_{4}$ spherical NPs. ${ }^{2}$ In a typical reaction, $0.174 \mathrm{~g}$ of $\mathrm{Fe}_{2} \mathrm{O}_{3}, 8 \mathrm{~mL}$ of OA, $20 \mathrm{~mL}$ of ODE were mixed in a three-neck flask and heated to $180^{\circ} \mathrm{C}$ and keep this temperature for 30 min with magnetic stirring under $\mathrm{N}_{2}$ flow. Then, the precursor solution was heated to $320{ }^{\circ} \mathrm{C}$ slowly and keep this temperature for 1 hour with magnetic stirring. The reaction solution is cooled to room temperature. Purification is conducted through addition of excess ethanol and centrifuging at $8000 \mathrm{rpm}$ for $2 \mathrm{~min}$. This method leads to $\mathrm{Fe}_{3} \mathrm{O}_{4}$ spherical nanocrystals coated OA ligands. The $\mathrm{Fe}_{3} \mathrm{O}_{4}$ spherical nanocrystals were redispersed in hexane.

Synthesis of $\mathrm{Fe}_{3} \mathrm{O}_{4}$ nanocubes. In a typical reaction, $0.261 \mathrm{~g}$ of $\mathrm{Fe}_{2} \mathrm{O}_{3}, 1.04 \mathrm{~g}$ of sodium oleate, $8 \mathrm{~mL}$ of OA, $20 \mathrm{~mL}$ of ODE were mixed in a three-neck flask and heated to $180{ }^{\circ} \mathrm{C}$ and were kept at this temperature for 30 min with magnetic stirring under $\mathrm{N}_{2}$ flow. Then, the precursor solution was heated to $320^{\circ} \mathrm{C}$ slowly and keep this temperature for 1 hour with magnetic stirring. The reaction mixture was cooled to room temperature. Purification was conducted through addition of hexane and excess ethanol and centrifuging at $8000 \mathrm{rpm}$ for $2 \mathrm{~min}$. The $\mathrm{Fe}_{3} \mathrm{O}_{4}$ nanocubes were redispersed in hexane.

Synthesis of $\mathbf{G d}_{2} \mathbf{O}_{3}$ nanoplatelets. The $\mathrm{Gd}_{2} \mathrm{O}_{3}$ nanoplatelets were prepared through a modified reaction. ${ }^{3}$ Lithium hydroxide $(6 \mathrm{mmol})$ was added into a mixture of $12 \mathrm{~mL}$ of OA, $18 \mathrm{~mL}$ of OAM, and $30 \mathrm{~mL}$ of ODE solvent and was heated to $110^{\circ} \mathrm{C}$. After evacuation at $110^{\circ} \mathrm{C}$ for $1 \mathrm{~h}$ to remove water, $3 \mathrm{mmol}$ of gadolinium acetate was added and the solution was evacuated for an extra hour at the same temperature. Then, the reaction solution was heated between $320^{\circ} \mathrm{C}$ for $1 \mathrm{~h}$ under high $\mathrm{N}_{2}$ blowing, resulting in the formation of the nanoplateletss. The reaction solution was cooled to room temperature. Purification was conducted through addition of excess methanol and centrifuging at 8000 rpm for $2 \mathrm{~min}$. The $\mathrm{Gd}_{2} \mathrm{O}_{3}$ nanoplatelets were redispersed in hexane.

Synthesis of CdSe nanorods. The CdSe nanorods (NRs) were prepared through a modified reaction. ${ }^{4}$ CdSe nanorods (NRs) capped with TOP, TOPO, TDPA and TBP were synthesized using a Schlenk- 
line. Typically, $210 \mathrm{mg}$ of CdO, $898 \mathrm{mg}$ of TDPA and $2.26 \mathrm{~g}$ of TOPO were mixed and heated to $120^{\circ} \mathrm{C}$ under vacumm for degassing. Then the mixture was heated to $320^{\circ} \mathrm{C}$ to form a transparent solution under $\mathrm{N}_{2}$ purge. After that, the Cd precursor was cooled to room temperature and aged for 12 h under $\mathrm{N}_{2}$. The $\mathrm{Cd}$ precursor was re-heated to $320^{\circ} \mathrm{C}$ after aging, while the Se precursor solution containing $62 \mathrm{mg}$ of Se powder, $1.44 \mathrm{~g}$ of TOP, $190 \mathrm{mg}$ of TBP was quickly injected. The solution was kept at $20 \mathrm{~min}$ for about $\mathrm{D}(3 \mathrm{~nm}) \times \mathrm{L}(10 \mathrm{~nm})$. The NRs was washed with ethanol and hexane for three times to remove the redundant ligands. A ligand exchange procedure was then performed, by adding DDT into the solutions with the same weight as NRs and stirring at $60^{\circ} \mathrm{C}$ for $12 \mathrm{~h}$. Finally, the NRs with DDT ligand were washed with ethanol and hexane for three times and stored in hexane.

Synthesis of Pt NPs. The Pt NPs were prepared through a modified reaction ${ }^{5} .150 \mathrm{mg}$ of the $\mathrm{Pt}(\mathrm{acac})_{2}$ were mixed in benzyl ether $(15 \mathrm{~mL})$ together with OAM $(1.23 \mathrm{~mL})$ and oleic acid $(2.39 \mathrm{~mL})$ in a threenecked flask. The mixture was evacuated at room temperature for 15 minutes, TOP ( $424 \mu \mathrm{L})$ was first added, then evacuated again and the mixture heated to $120^{\circ} \mathrm{C}$. The solution was left under vacuum for $30 \mathrm{~min}$ to remove all water and other impurities. At this point, pale yellow transparent solutions were obtained. The reaction flask was then flushed again with nitrogen and heated very quickly $\left(\sim 40^{\circ} \mathrm{C}\right.$ $\min ^{-1}$ ) to $290^{\circ} \mathrm{C}$. After 15 minutes of reaction at the appropriate temperature and magnetic stirring, the solution was allowed to cool to room temperature. The Pt NPs were isolated by addition of isopropanol and centrifugation (8000 rpm, $3 \mathrm{~min}$ ) and redispersed in $n$-hexane.

Synthesis of Pd NPs. The Pd NPs were prepared through a modified reaction ${ }^{5} .150 \mathrm{mg}$ of the $\mathrm{Pd}(\mathrm{acac})_{2}$ were mixed in benzyl ether $(15 \mathrm{~mL})$ together with oleylamine $(1.23 \mathrm{~mL})$ in a three-necked flask, the mixture was evacuated at room temperature for 15 minutes, the flask flushed with nitrogen and TOP $(424 \mu \mathrm{L})$ was first added, then evacuated again and the mixture heated to $120^{\circ} \mathrm{C}$. The solution was left under vacuum for $30 \mathrm{~min}$ to remove all water and other impurities. At this point, pale yellow transparent solutions were obtained. The reaction flask was then flushed again with nitrogen and heated very quickly $\left(\sim 40^{\circ} \mathrm{C} \mathrm{min}^{-1}\right)$ to $270{ }^{\circ} \mathrm{C}$. After 15 minutes of reaction at the appropriate temperature and magnetic stirring, the solution was allowed to cool to room temperature. The Pd NPs were isolated by addition of isopropanol and centrifugation ( $8000 \mathrm{rpm}, 3 \mathrm{~min})$ and redispersed in $n$-hexane.

Synthesis of $\mathrm{Co}(\mathrm{OH})_{2}$ NTs. The $\mathrm{Co}(\mathrm{OH})_{2}$ NTs were prepared through a modified reaction ${ }^{6} .0 .056 \mathrm{~g}$ of $\mathrm{CoSO}_{4} \cdot 7 \mathrm{H}_{2} \mathrm{O}$ and $0.012 \mathrm{~g}$ of trimethyltetradecylammonium chloride (TTAC) were added to a 20 $\mathrm{mL}$ Teflon-lined autoclave, followed by the addition of $1 \mathrm{~mL}$ octylamine and $18 \mathrm{~mL}$ hexane in sequence. The octylamine was stored at $40^{\circ} \mathrm{C}$ and the precursors' colour in autoclave immediately changed to blue when octylamine was injected. After sonicating for several minutes, the autoclave was sealed and heated at $142^{\circ} \mathrm{C}$ for $10 \mathrm{~h}$. The autoclave was cooled to room temperature. Purification was 
conducted through addition of excess ethanol and centrifuging at $6000 \mathrm{rpm}$ for $2 \mathrm{~min}$. The $\mathrm{Co}(\mathrm{OH})_{2}$ NTs were redispersed in hexane.

Assembly of Au NPs within NTs (Au/NTs). $0.5 \mathrm{~mL}$ of $n$-hexane solution including $0.5 \mathrm{mg}$ of $\mathrm{Co}(\mathrm{OH})_{2} \mathrm{NTs}$ and $0.5 \mathrm{mg}$ of Au NPs (Au3, Au4, Au5, Au6, Au 7 or Au13) were added into 2-mL vial, followed by placing the vial into $20-\mathrm{mL}$ glass containing $2 \mathrm{~mL}$ of ethanol. The vial was sealed with a polypropylene cap lined with silicone rubber, followed by subjecting to $50^{\circ} \mathrm{C}$ for 8 hours. After that, the precipitate was separated by centrifugation and then redisperse in ethanol (see Figure S1).

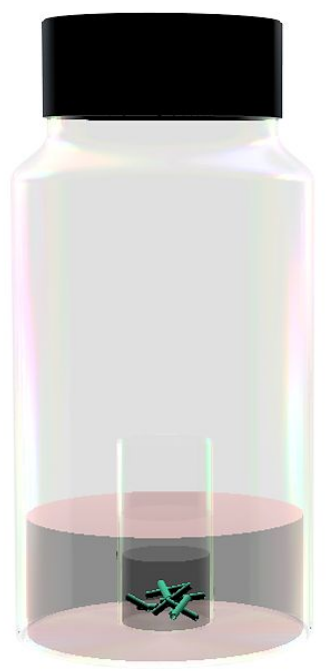

Figure S1. Scheme of the vial-in-vial setup for the assembly experiment.

Assembly of other nanocrystals (NCs), including $\mathrm{Fe}_{3} \mathrm{O}_{4} \mathrm{NPs}, \mathrm{Fe}_{3} \mathrm{O}_{4}$ nanocubes, $\mathrm{Gd}_{2} \mathrm{O}_{3}$ nanoplatelets, CdSe nanorods, Pt NPs and Pd NPs were carried out with similar procedures as described above, with diverse amount of NPs as described in Table S2.

Assembly of Au NPs at the external surface of NTs (Au@NTs). $0.5 \mathrm{mg}$ of $\mathrm{Co}(\mathrm{OH})_{2}$ NTs and 0.5 mg of Au NPs (Au3, Au4 or Au5) were dispersed in $0.5 \mathrm{~mL}$ of hexane containing $10 \mu \mathrm{L}$ of (-)limonene in a $2-\mathrm{mL}$ glass vial, followed by placing the vial into $20-\mathrm{mL}$ glass containing $2 \mathrm{~mL}$ of ethanol. The vial was sealed with a polypropylene cap lined with silicone rubber, followed by subjecting to $50^{\circ} \mathrm{C}$ for 8 hours. After that, the precipitate was separated by centrifugation and then redisperse in ethanol. 
Table S2. Reaction conditions used in this work for the preparation of the different NPs superlattices within the $\mathrm{Co}(\mathrm{OH})_{2}$ NTs.

\begin{tabular}{cc}
\hline Samples & $\mathbf{m}_{\mathbf{N P}} / \mathbf{m}_{\mathbf{N T s}}$ \\
\hline $\mathrm{Fe}_{3} \mathrm{O}_{4}$ nanoparticles & 0.2 \\
$\mathrm{Fe}_{3} \mathrm{O}_{4}$ nanocubes & 0.2 \\
$\mathrm{Gd}_{2} \mathrm{O}_{3}$ nanoplatelets & 0.3 \\
$\mathrm{Pt}$ nanoparticles & 0.6 \\
$\mathrm{Pd}$ nanoparticles & 0.5 \\
$\mathrm{CdSe}$ nanorods & 0.2 \\
\hline
\end{tabular}

Characterization techniques. Detailed morphological and structural characterizations were carried out by using a transmission electron microscope (TEM) (JEM 1011 and JEM 2100). Elemental characterization of the samples including HAADF and EDS images were carried out by using STEM (FEI Tecnai G2 TF20). The Fourier Transform infrared (FTIR) spectra were recorded on the Bruker INVENIO FTIR spectrometer with $\mathrm{KBr}$ pellets. UV-Vis absorption spectra of the samples were obtained by a SIMADAZU UV-3150 UV-Vis spectrometer with quartz cells. ${ }^{1} \mathrm{H}-\mathrm{NMR}$ spectra were recorded on a $400 \mathrm{MHz}$ Bruker Avance spectrometer. The surface area measurements were performed with $\mathrm{N}_{2}$ adsorption/desorption isotherms at liquid nitrogen temperature $(77 \mathrm{~K})$ using a QuadraSorb SI. The yield of catalytic alcoholysis of silanes and semihydrogenation of alkynes to alkenes were determined by Gas chromatography-mass spectra (GC-MS, 8860 GC System, 59778 GC/MSD). Measured the mass of $\mathrm{Co}(\mathrm{OH})_{2}$ nanotubes as a function of temperature by The thermogravimetric analysis (TGA, TGA5500).

Catalytic alcoholysis of silanes. Au Catalysts $(0.1 \mathrm{mg})$ was loaded into a mixed solution of alcohol $(5.0 \mathrm{~mL})$ and silane $(0 \sim 10 \mathrm{mmol})$ and the reaction mixture was stirred at $30^{\circ} \mathrm{C}$. The yield was determined by GC-MS analysis. All of the reaction yields reported were averaged values from three independent measurements.

Semihydrogenation of alkynes to alkenes. Pd Catalysts $(0.1 \mathrm{mg})$ was loaded into a mixed solution containing $10 \mathrm{~mL}$ of acetone, $5 \mathrm{mmol}$ of alkynes, $10 \mathrm{mmol}$ of silane and $10 \mathrm{mmol}$ of DIPEA. The mixture was stirred in a temperature-fixed water bath $\left(30^{\circ} \mathrm{C}\right)$ for a certain period. The yield was determined by GC-MS. All of the reaction yields reported were averaged values from three 
independent measurements.

\section{Supplementary references}

1 Goubet, N.; Portalès, H.; Yan, C.; Arfaoui, I.; Albouy, P.-A.; Mermet, A.; Pileni, M.-P. Simultaneous Growths of Gold Colloidal Crystals. J. Am. Chem. Soc. 2012, 134, 3714-3719.

2 Park, J.; An, K. J.; Hwang, Y. S.; Park, J. G.; Noh, H. J.; Kim, J. Y.; Park, J. H.; Hwang, N. M.; Hyeon, T. Ultra-large-scale syntheses of monodisperse nanocrystals. Nat. Mater. 2004, 3, 891895.

3 Paik, T.; Gordon, T. R.; Prantner, A. M.; Yun, H.; Murray C. B. Designing tripodal and triangular gadolinium oxide nanoplates and self-assembled nanofibrils as potential multimodal bioimaging probes. ACS Nano 2013, 7, 2850-2859.

4 Peng, Z.; Peng. Xiao. Nearly monodisperse and shape-controlled CdSe nanocrystals via alternative routes: nucleation and growth. J. Am. Chem. Soc. 2002, 124, 3343-3353.

5 Cargnello, Matteo.; Doan-Nguyen, V. V. T.; Gordon, T. R.; Diaz, R.; Stach, E. A.; Gorte, R. J.; Fornasiero, P.; Murray, C. B. Control of metal nanocrystal size reveals metal-support interface role for ceria catalysts. Science 2013, 341, 771-773.

6 Ni, B.; Liu, H.; Wang P-p.; He, J.; Wang, X. General synthesis of inorganic single-walled nanotubes. Nat. Commun. 2015, 6, 8756 . 


\section{Molecular dynamics simulations.}

United-atom MD simulations were applied in the current simulation. The original coordinates for methanol, dodecanethiol, and diphenylsilane (DPS) were taken from the automated force field topology builder (ATB) with optimized geometry. Bonded and non-bonded parameters were obtained from the GROMOS 54a7 parameter set. In order to realistically simulate the internal environment of the open porous NP superlattices with $C 4$ symmetry, the structure here was composed of four $\mathrm{Au}$ domes (geometric curvature $\kappa=0.5 \mathrm{~nm}^{-1}$ ) in square arrangement and was kept at fixed position during the whole simulation. The space in the $C 4 N P$ superlattices can be regarded as a channel with a diameter of $\sim 4 \mathrm{~nm}$. First, the dodecanethiol ligands were randomly arranged on the surface of the $\mathrm{Au}$ domes with a packing density of 4.6 ligands per $\mathrm{nm}^{2}$ by Packmol program and the initial structure was placed in the center of a cubic box with a size of $10 \times 10 \times 15 \mathrm{~nm}^{3}$. Methanol molecules were used as a solvent to fill the entire box and 10 DPS molecules were randomly distributed in the system. Subsequently, the steepest descent method was used until the maximum forces converged below 100 $\mathrm{kJ} \mathrm{mol}^{-1} \mathrm{~nm}^{-1}$. Then, $1 \mathrm{~ns}$ NPT simulation was performed at $1 \mathrm{~atm}$ and $298 \mathrm{~K}$ to keep the system in the appropriate volume. Finally, 20 ns NVT simulation was executed at $298 \mathrm{~K}$ to get MD trajectories. In the simulation, periodic boundary conditions were used in all directions, the LINCS algorithm was applied to constrain the bond lengths, and a time step of $2 \mathrm{fs}$ was set. A cutoff distance $1.2 \mathrm{~nm}$ was used for Lennard-Jones interactions. Electrostatic interactions were calculated using the Particle Mesh Ewald (PME) algorithm. The number of molecules used in the system were given in detail in the Table S3.

Table S3. Detailed information on the number of molecules for the simulation system.

\begin{tabular}{ccllc}
\hline N(Au) & $N$ (dodecanethiol) & $N$ (methanol) & $N($ DPS $)$ & Box dimensions / nm \\
\hline 5706 & 580 & 8500 & 10 & $10 \times 10 \times 15$ \\
\hline
\end{tabular}




\section{Supplementary Note 1 . Alkyl chain density on $\mathrm{Co}(\mathrm{OH})_{2}$ NTs}

We have performed TGA measurements for $\mathrm{Co}(\mathrm{OH})_{2}$ NTs samples to determine the alkyl chain density on the surfaces. First, we assume that the density of octylamine coated on the external and internal surface is the same. Therefore, we are able to obtain the amount the octylamine coated on NTs when they are burning out at elevated temperature $\left(>450^{\circ} \mathrm{C}\right)$. From the TGA curve in Figure S2, one can found a continuous weight loss of $\sim 37 \%$ in the range of $25-450^{\circ} \mathrm{C}$, which can be categorized as two main processes: (1) loss of adsorbed water in the range of $25-130^{\circ} \mathrm{C}$, which is determined to be $\sim 6 \%$; (2) In the range of $130-450^{\circ} \mathrm{C}$, dehydration of $\mathrm{Co}(\mathrm{OH})_{2}$, followed by the oxidation to $\mathrm{Co}_{3} \mathrm{O}_{4}$ is most probable, which is also accompanied by the burning of organic ligands, which comprises of $\sim 31 \%$. As a result, the organics can be determined to be $\sim 22 \%$, Hence the surface ligand density can be calculated to be 2.4 octylamine per $\mathrm{nm}^{2}$.

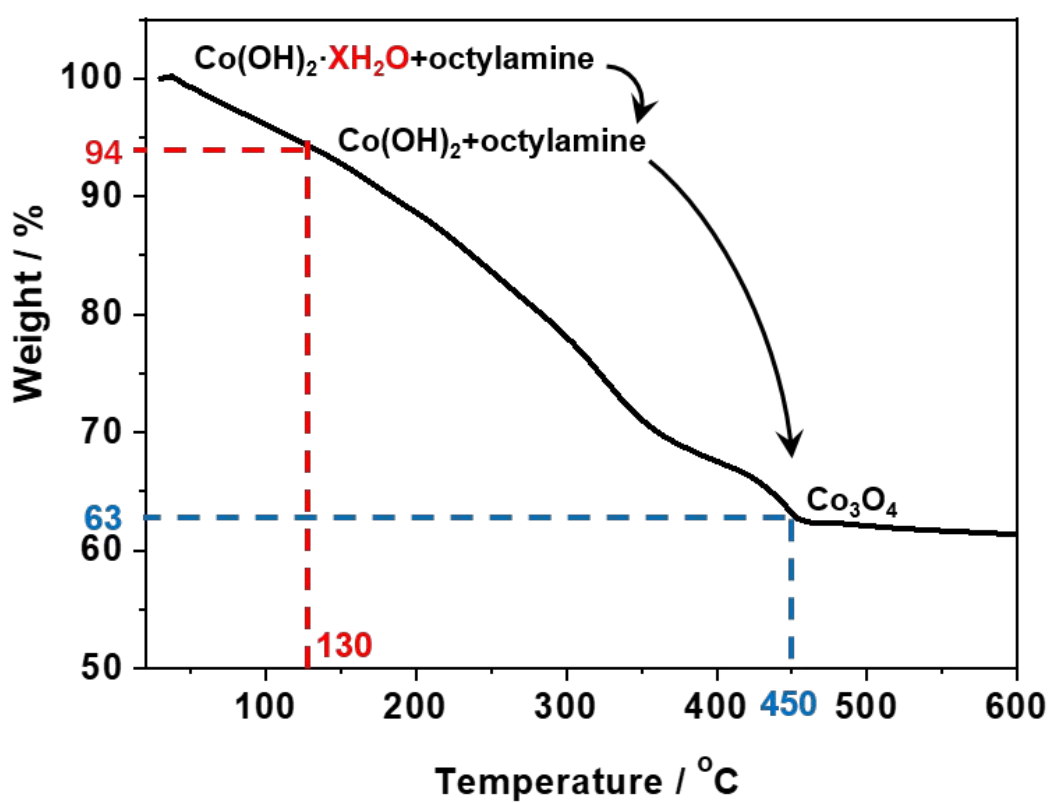

Figure S2. TGA curve of $\mathrm{Co}(\mathrm{OH})_{2}$ NTs from room temperature to $600^{\circ} \mathrm{C}$. 
The quantified numbers for the dependence of ligand density with distance from surface is from theoretical calculations, which presents in Figure 1e in main text. The details can be found as follows: Ligand packing density $\left(\lambda_{0}\right)$ is defined as:

$$
\lambda_{0}=\frac{A_{c}}{A_{L}}(\mathrm{~S} 1)
$$

Where $A_{c}$ denotes the cross-sectional area of the aliphatic chain when they are close-packed, and $A_{L}$ the available surface area per ligand on the grafting surface. The $A_{c}$ can be estimated from a closepacking separation distance of $\sim 0.5 \mathrm{~nm}$, resulting in a $A_{c} \approx 0.2 \mathrm{~nm}^{2}$; the $A_{L}$ on the surface of nanotubes is calculated from the TGA data $\left(\sim 2.4\right.$ octylamine per $\left.\mathrm{nm}^{2}\right)$, resulting in $A_{L} \approx 0.42 \mathrm{~nm}^{2}$. The above data leads to $\lambda_{0}=0.48$ at both internal and external surface of the nanotube. In the case of the external surface with positive curvature, the molecular packing diverges and the distance between two neighboring molecules increases remarkably in the tail region. The $\lambda_{0}(d)$ decreases with increasing distance from the external nanotube surface, $d$, and can be expressed as:

$$
\lambda_{0}(d)=\frac{r_{e x t}}{r_{e x t}+d} \lambda_{0}
$$

Where $r_{\text {ext }}$ is the external radius of the nanotube, which can be calculated from the sum of the internal radius $\left(r_{i n t}=9.3 \mathrm{~nm}\right)$ and the thickness of the tube wall $(1 \mathrm{~nm})$.

On the contrary, for internal surface with negative curvature, the molecular packing converges and the distance between two neighboring molecules decreases in the tail region. The $\lambda_{0}(d)$ increases with increasing distance from the internal nanotube surface, $d$, and can be expressed as:

$$
\lambda_{0}(d)=\frac{r_{\text {int }}-L}{r_{\text {int }}-d} \lambda_{0}^{\prime}
$$

Where $L$ is the molecule length of octylamine, which is around $1 \mathrm{~nm} ; \lambda_{0}^{\prime}$ is the ligand packing density at the tail end, which can be calculated when $d=0, \lambda_{0}^{\prime} \approx 0.54$.

In the both conditions, $0 \leq d \leq L$. 


\section{Supplementary Note 2. Interaction energy between NP and NT.}

For the modeling of the interaction energy between NPs and NTs, two scenarios were considered: one is the NP contacts with the external surface of the NT, the other one is the NP contacts with the internal surface of the NT. The main attractive force is the van der Waals interactions from the chain-chain interactions. Assuming that two mutually paralleled alkyl chains have maximized van der Waals forces, as indicated by Salem, the attractive interactions between NP and NT can be estimated from the pairs of alky chains that are parallel arranged. For simplicity, the n-dodecyl (C12) alkyl chain grafting density for both NT and NP is 4 chains per $\mathrm{nm}^{2}$. Hence each chain occupies $0.25 \mathrm{~nm}^{2}$.

a

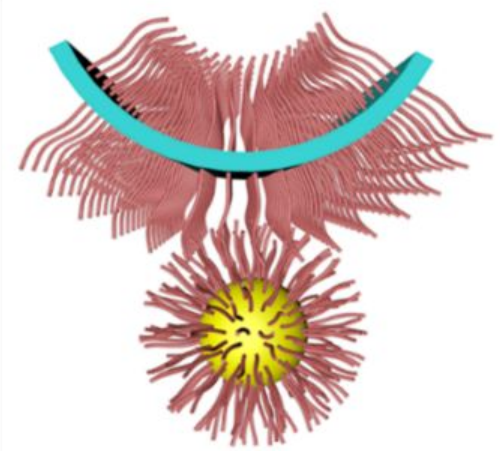

OPM model b

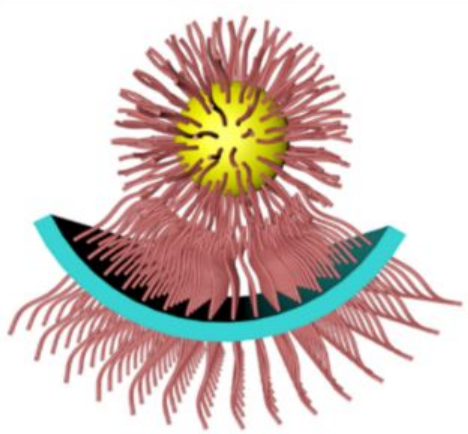

Maximized pairs of paralleled alkyl chains

Figure S3. (a) NP interact with the external surface of the NT follows the OPM model; (b) NP interact with the internal surface of the NT would maximize the pairs of paralleled alkyl chains, which leads to the maximization of van der Waals attractive energy.

(1) NP at external surface of NT. The interaction energy between NP and NT can be estimated by optimal packing model (OPM), which assumes that the ligands lying on the surface-surface line pack densely within a narrow volume (Figure S3a). To the first assumption, the interaction energy can be estimated by two paralleled $\mathrm{C} 12$ chains. The van der Waals attraction $\left(U_{C 12}\right)$ between two nearest parallel alkyl chains of length $L$ from $N$ identical basic units $(L=N \lambda)$ and separated by a distance $\mathrm{D}$ has been given by Salem:

$$
U_{C 12}=\mathrm{A} \frac{3 \pi L}{8 \lambda^{2} D^{5}}
$$

Where $A$ is the Hamaker constant of methylene units. With Salem's conclusion that the attractive energy is correlated with the length of the alkyl chain, and with the attractive energy for C12 is calculated to be $-9.6 \mathrm{k}_{\mathrm{B}} \mathrm{T} /$ molecule, we could relate the overlapping length and interaction strength: 


$$
U_{a t t r} \approx\left(-4.8 k_{B} T\right) \times(2 L-d)
$$

Where $d$ is the distance between two surfaces. The elastic repulsion energy between two C12 chains can be calculated on the basis of the elastic modulus $(E)$, which is on the order of $\sim 0.86 \mathrm{GPa}$ for dodecyl alkyl chains. Hence the elastic repulsion energy can be estimated to be:

$$
U_{e l} \approx \frac{1}{2} \times \frac{E A_{0}}{L} \approx\left(17.2 k_{B} T\right) \times(2 L-d)^{2}(\mathrm{~S} 6)
$$

Where $A_{0}$ is the cross-sectional area of an alkyl chain $\left(\mathrm{A}_{0} \approx 0.25 \mathrm{~nm}^{2}\right)$. Considering the attractive and elastic repulsive energy between two parallel $\mathrm{C} 12$ chains, we claim that the overall contribution from the ligand-ligand interactions can be less than $-k_{B} T$.

(2) NP at internal surface of NT. In this case, we found that nearly all the alkyl chains between NP and NT were paralleled when they are approaching to each other (Figure S3b). Hence the total energy between two surfaces can be estimated from the following equation:

$$
U_{N P-N T} \approx S \rho_{C 12}\left[\left(-4.8 k_{B} T\right) \times(2 L-d)+\left(17.2 k_{B} T\right) \times(2 L-d)^{2}\right](\mathrm{S} 7)
$$

Where $S$ is the total contacting areas between the NT and NP surfaces. With this simple model, we found that the interaction energy between NP and NT can be tens of $\mathrm{k}_{\mathrm{B}} \mathrm{T}$, one or two orders of magnitude larger than that NP at internal surface of NT. 


\section{Supplementary Note 3.2D close-packing of small circular disks inside a large circular disk.}

The porosity of these NP/NTs nanocomposites is mainly induced by the geometric packing of NPs within the NTs, which can be further simplified as the circular packing problem. The circular packing problem deals with the $2 \mathrm{D}$ arrangement of $N$ identical small circular disk (diameter $\mathrm{d}_{\mathrm{S}}$ ) in a larger circular disk (diameter, $d_{L}$ ), which is closely associated with the ratio $d_{L} / d_{S}$. When $d_{L} / d_{S}=1$, it forms structure with $C 1$ symmetry. When $\mathrm{d}_{\mathrm{L}} / \mathrm{d}_{\mathrm{S}}>1$, it can be expressed as follows:

$$
d_{L}(N)=d_{S}\left(1+\frac{1}{\sin \pi / N}\right)
$$

Where $\quad d_{L}(2)=2 d_{S}, \quad d_{L}(3)=2.155 d_{S}, \quad d_{L}(4)=2.414 d_{S}, \quad d_{L}(5)=2.701 d_{S}, \quad d_{L}(6)=2.873 d_{S}$, which results in $C 2, C 3, C 4, C 5$ and $C 6$ symmetric structures as shown in Figure S4.

The porosity in $\mathrm{C} 1$ and $\mathrm{C} 2$ symmetric structures can be related to the voids between NPs and NTs. While for the porosity in C4 and C5 symmetric structures, the porosity can be related to two facts: (1) voids between NPs and NTs; (2) the central voids from the NPs packing. When $d_{L} / d_{S} \geq 2.873$, NPs within the NTs will adopt the hexagonal close packing mode, leading to nanocomposites with low porosity.
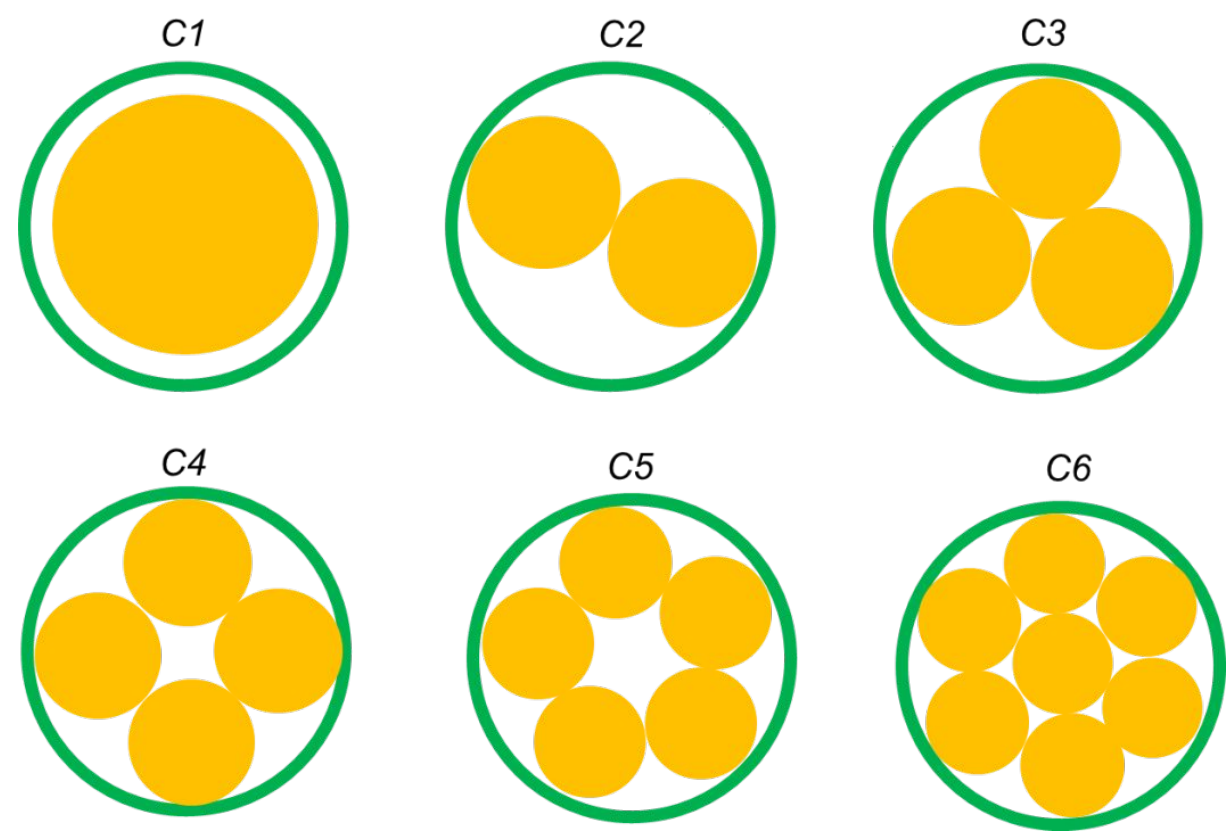

Figure S4. Circle packing geometry with six solutions. 


\section{Supplementary Note 4. Supramolecular vessels from self-assembled alkyl chains at the internal and external surfaces of NTs.}

The supramolecular vessels are built on the basis of the respective "divergent" and "convergent" features for self-assembled octylamine at the external and internal surfaces, which result in the formation of "jar" and "Erlenmeyer flask" with wide and narrow mouth, respectively. Assuming that the grafting density of octylamine molecules on NTs is identical throughout the surface $(2.4$ octylamines per $\mathrm{nm}^{2}$ ), two different flask models can be depicted in Figure S5. For the "jar" with a wide mouth, the diameter and surface area of the mouth is $0.81 \mathrm{~nm}$ and $0.5 \mathrm{~nm}^{2}$, respectively, whereas it is $0.64 \mathrm{~nm}$ and $0.33 \mathrm{~nm}^{2}$ for the "Erlenmeyer flask". The height of these two supramolecular vessels is both $\sim 1 \mathrm{~nm}$ from $\mathrm{C} 8$ chains.

The size of limonene molecule is $1.04 \times 0.67 \times 0.54 \mathrm{~nm}^{3}$, which is quite close to that of supramolecular flask at the internal surface of the NTs. Once these limonene molecules are encapsulated into the supramolecular flask, they are likely to be trapped, as evidenced by the NMR and CD spectra (Figure $3 b-3 c$ in the main text). While for supramolecular vessel (jar-like) at the external surface of NTs, these limonene molecules are unlikely to be trapped since the size of the jar" like vessel is much larger than of that of limonene molecule. When the internal surface of NTs was supramolecularly occupied by the molecules, like limonene or pinene with proper sizes, the chain-chain van der Waals interactions between NTs and NPs will be inhibited, which leads to the assembly of NP at the external surface of NTs. 
a

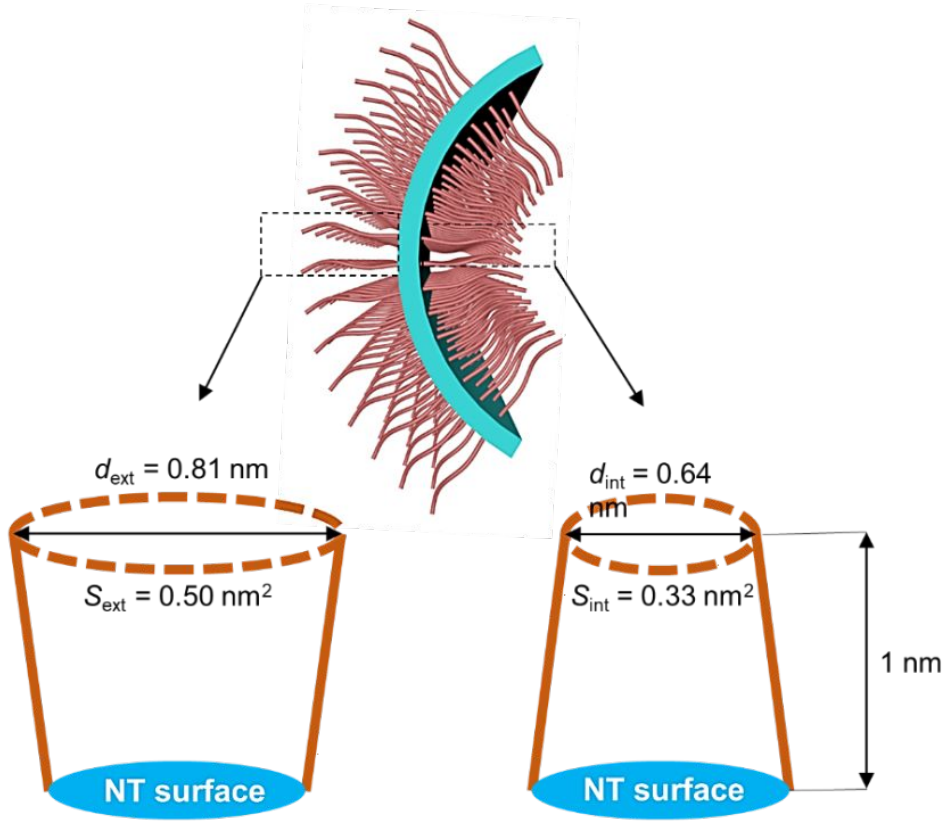

“jar" like vessel

b

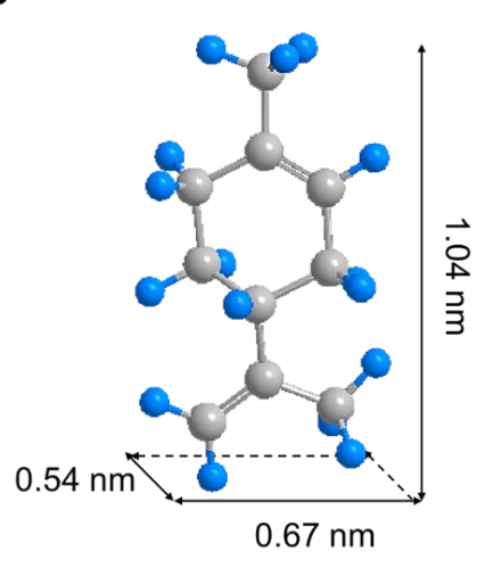

Limonene "flask" like vessel

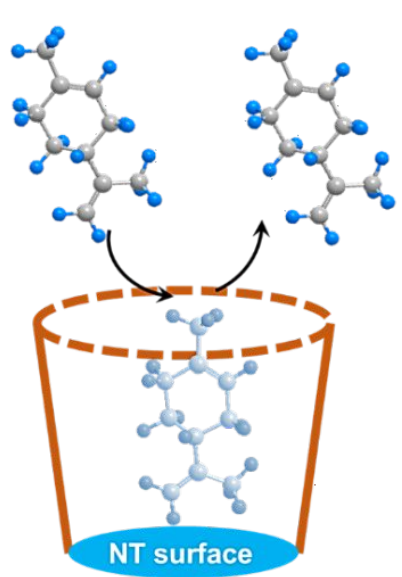

NT surface

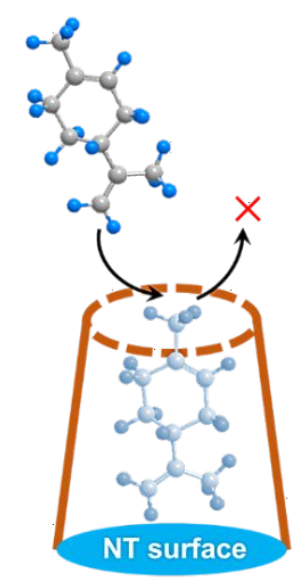

Supramolecularly trapped

Figure S5. (a) Supramolecular vessel models of the self-assembled alkyl chains on external or internal surface of NTs. (b) supramolecularly trapped limonene molecule within the supramolecular vessel at the internal surface of NTs. 


\section{Supporting figures.}
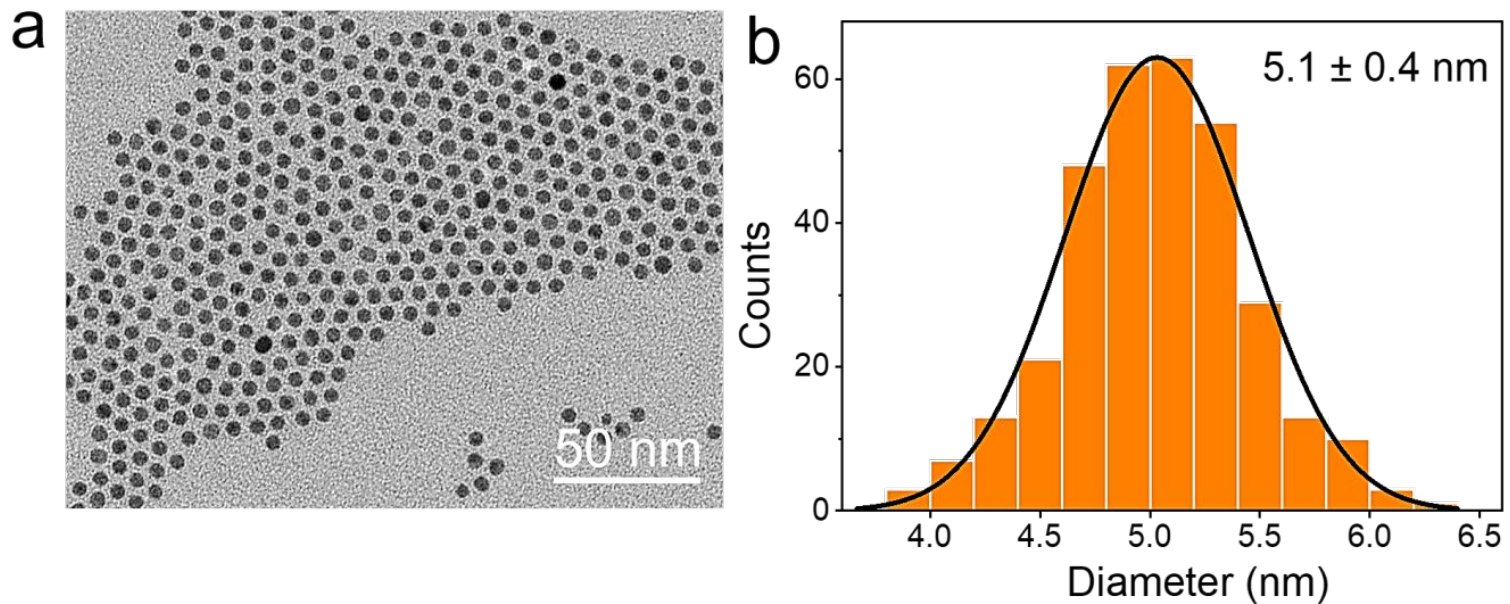

C

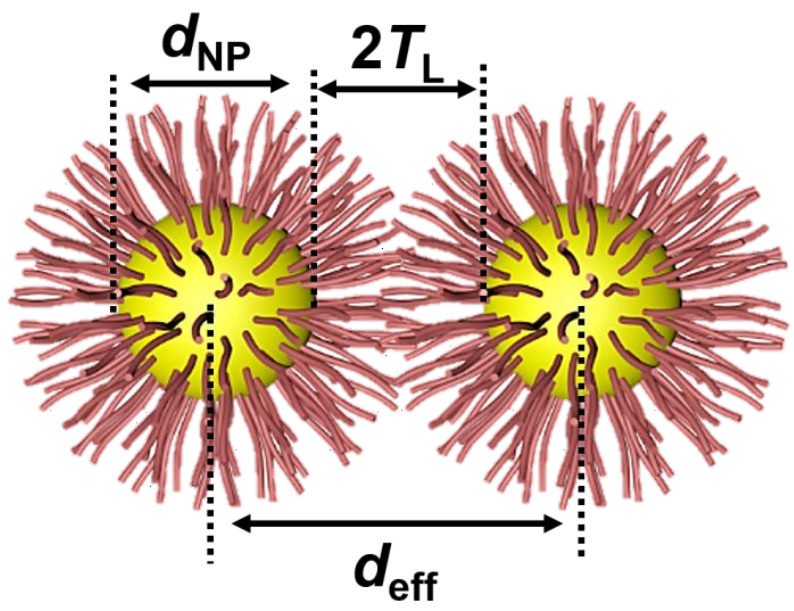

Figure S6. Representative TEM image (a) and size histograms (b) of Au5 NPs capped with dodecanethiol. (c) Schematic diagram of the effective diameter $\left(d_{\mathrm{eff}}\right)$ of a NP is determined by the sum of metal core diameter $\left(d_{\mathrm{NPs}}\right)$ and twice the thickness of the organic ligand $\left(2 T_{\mathrm{L}}, \sim 2 \mathrm{~nm}\right)$. 

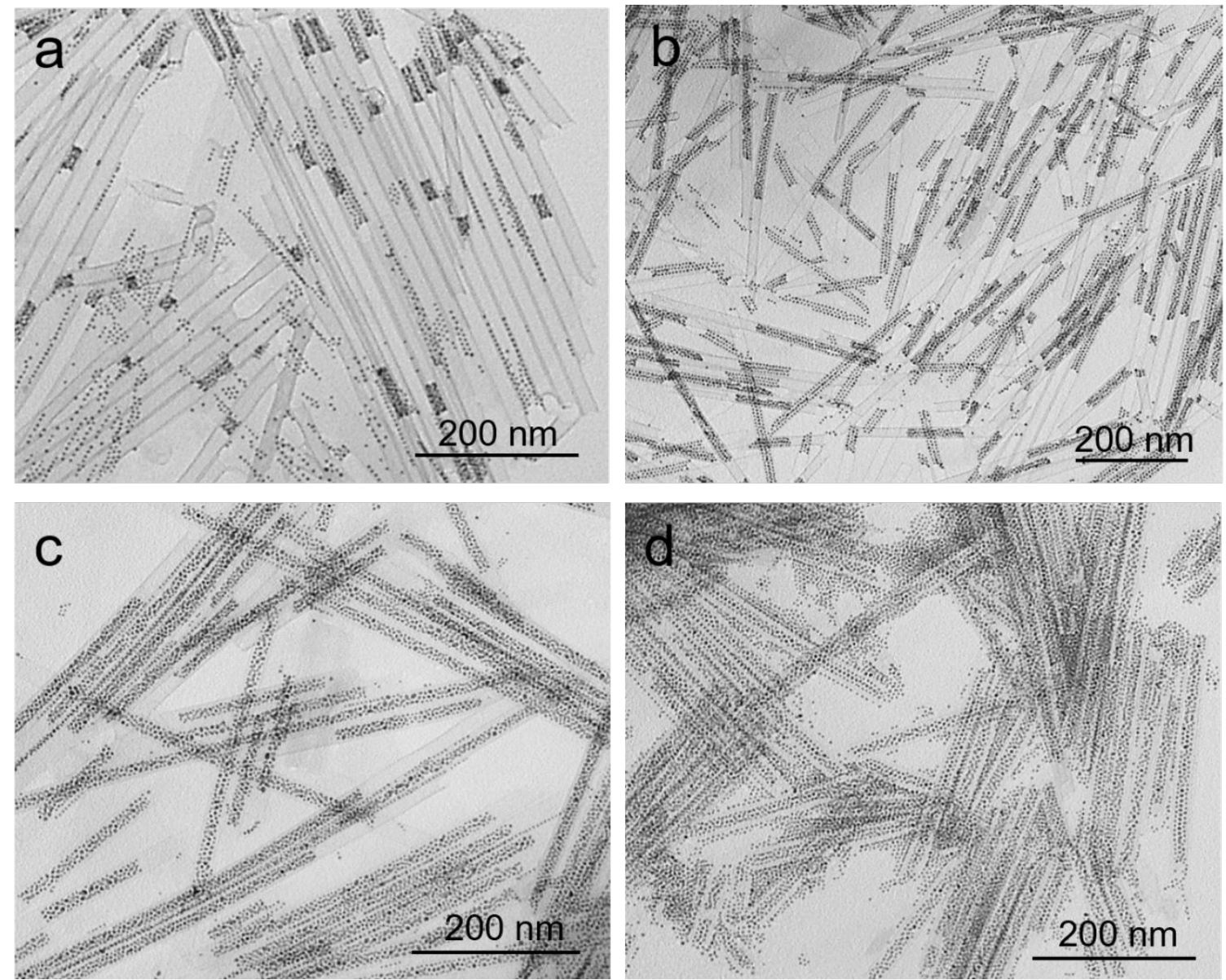

Figure S7. TEM images of Au5 NPs assembly inside the $\mathrm{Co}(\mathrm{OH})_{2}$ NTs with different $\alpha\left(\alpha=\mathrm{m}_{\mathrm{Au}} / \mathrm{m}_{\mathrm{NTs})}=0.1\right.$ (a), $\alpha=0.5$ (b), $\alpha=1$ (c), $\alpha=2$ (d). 

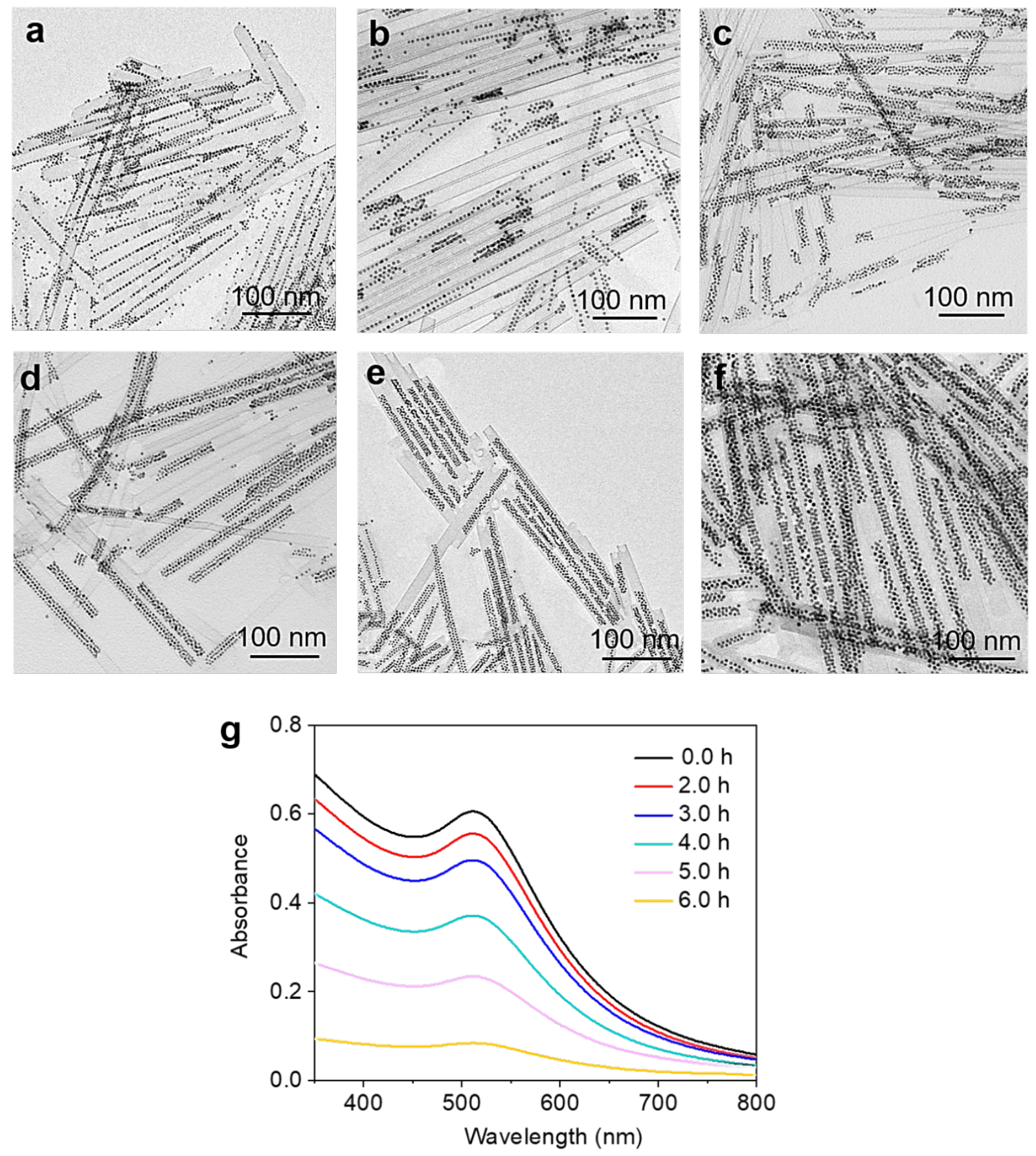

Figure S8. TEM images of Au5 NPs assembly inside the $\mathrm{Co}(\mathrm{OH})_{2}$ NTs with different reaction time, $0 \mathrm{~h}(\mathbf{a}), 2 \mathrm{~h}(\mathbf{b}), 3 \mathrm{~h}(\mathbf{c}), 4 \mathrm{~h}(\mathbf{d}), 5 \mathrm{~h}(\mathbf{e}), 6 \mathrm{~h}(\mathbf{f})$. Time resolved UV-Vis spectra of diluted supernatant , which reveal that Au5 NPs were gradually precipitating out of hexane (g). 

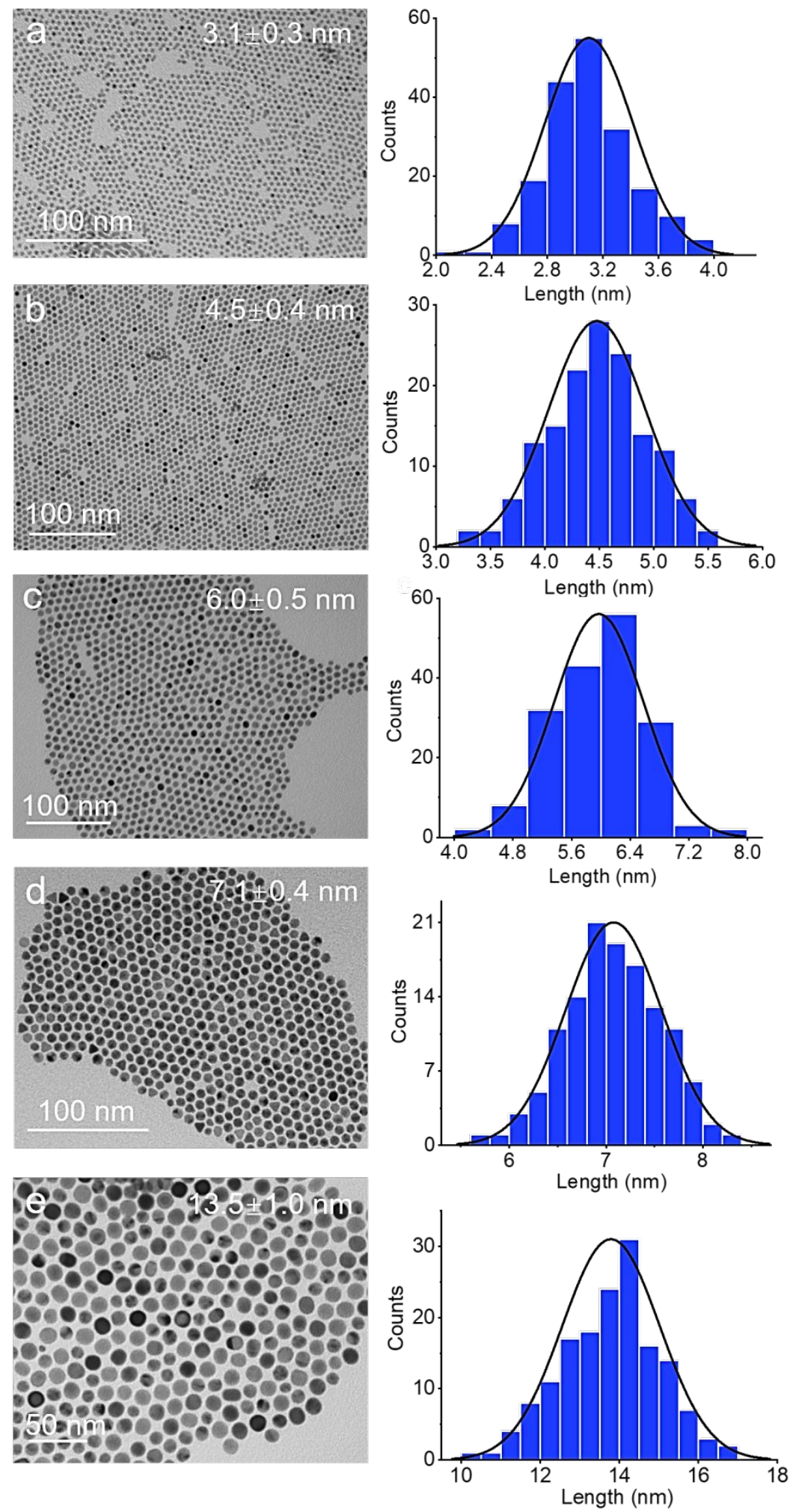

Figure S9. Representative TEM images of Au NPs capped with 1-dodecanethiol with different diameters. For Au13 NPs, they are capped with oleylamine. The right part is the corresponding size histograms measured from 300 NPs. 


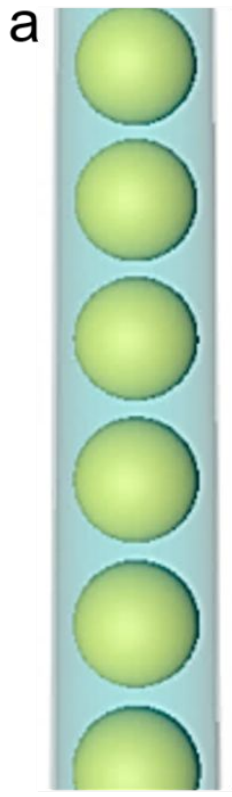

C1
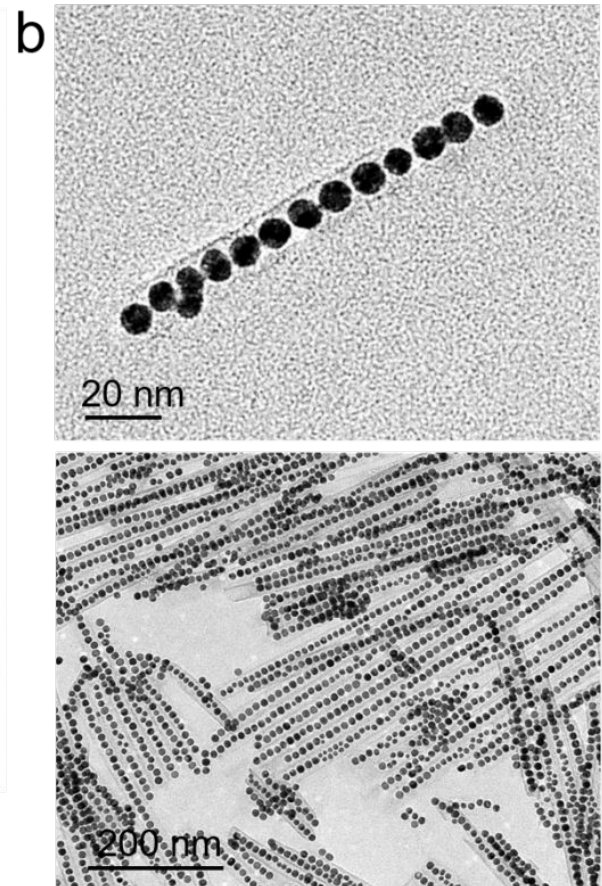
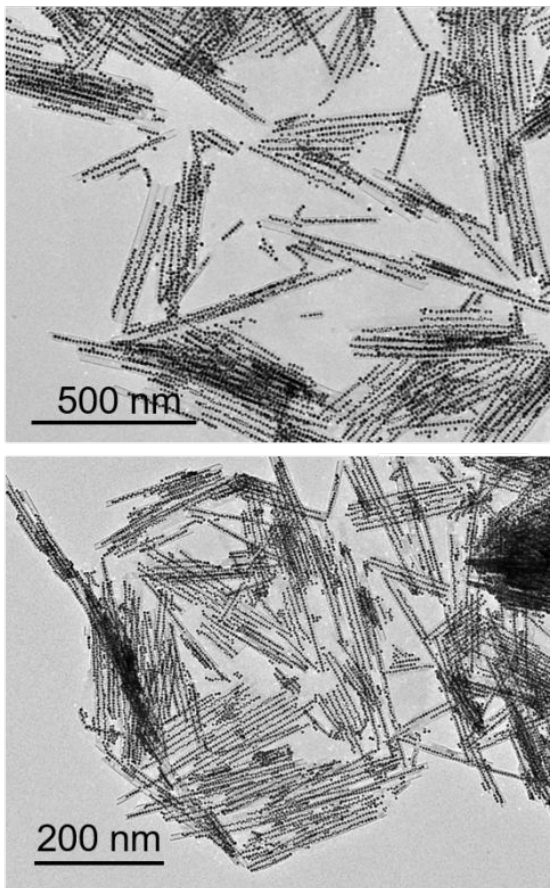

Figure S10. The model (a) and the corresponding TEM images (b) of Au13/NTs.

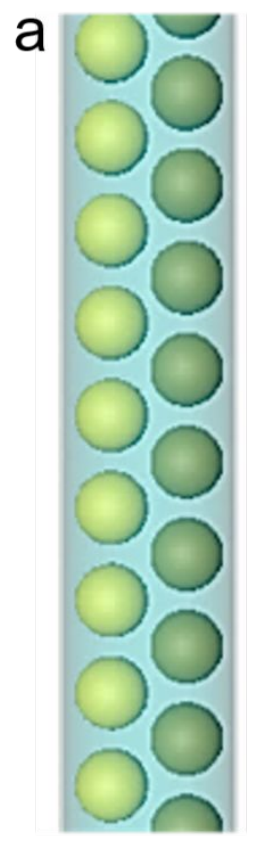

zigzag
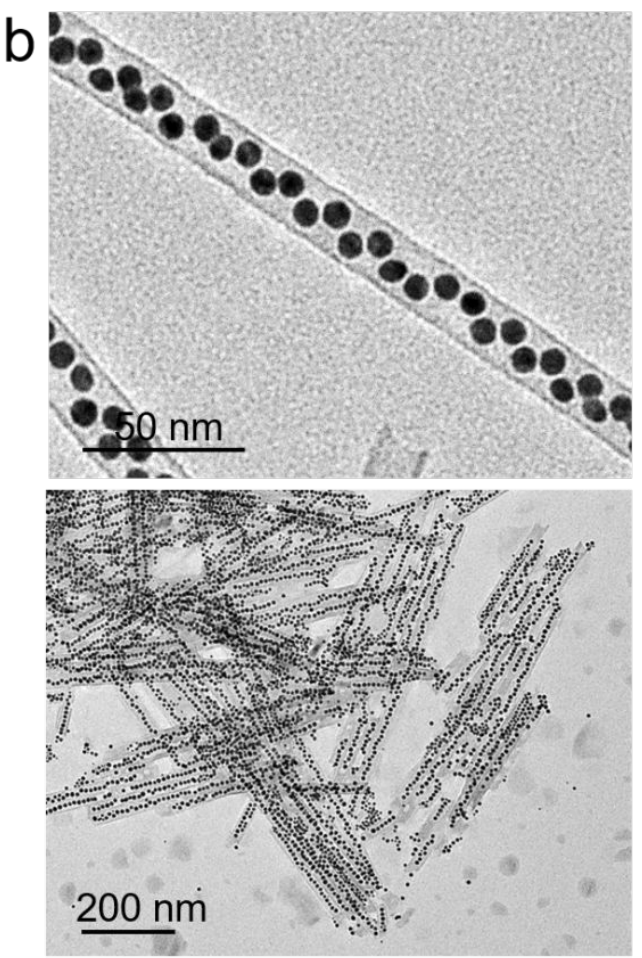
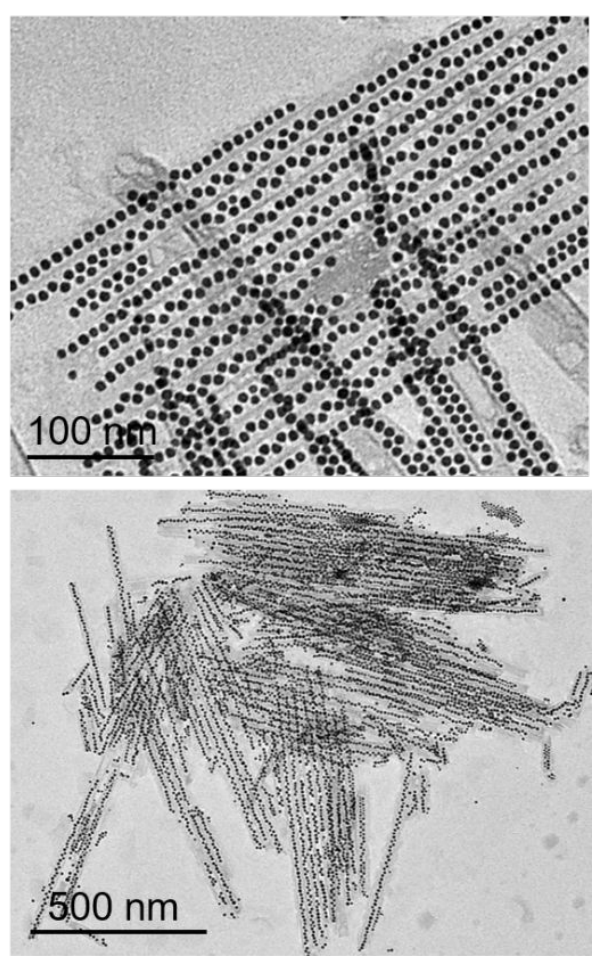

Figure S11. The model (a) and the corresponding TEM images (b) of Au7/NTs. 

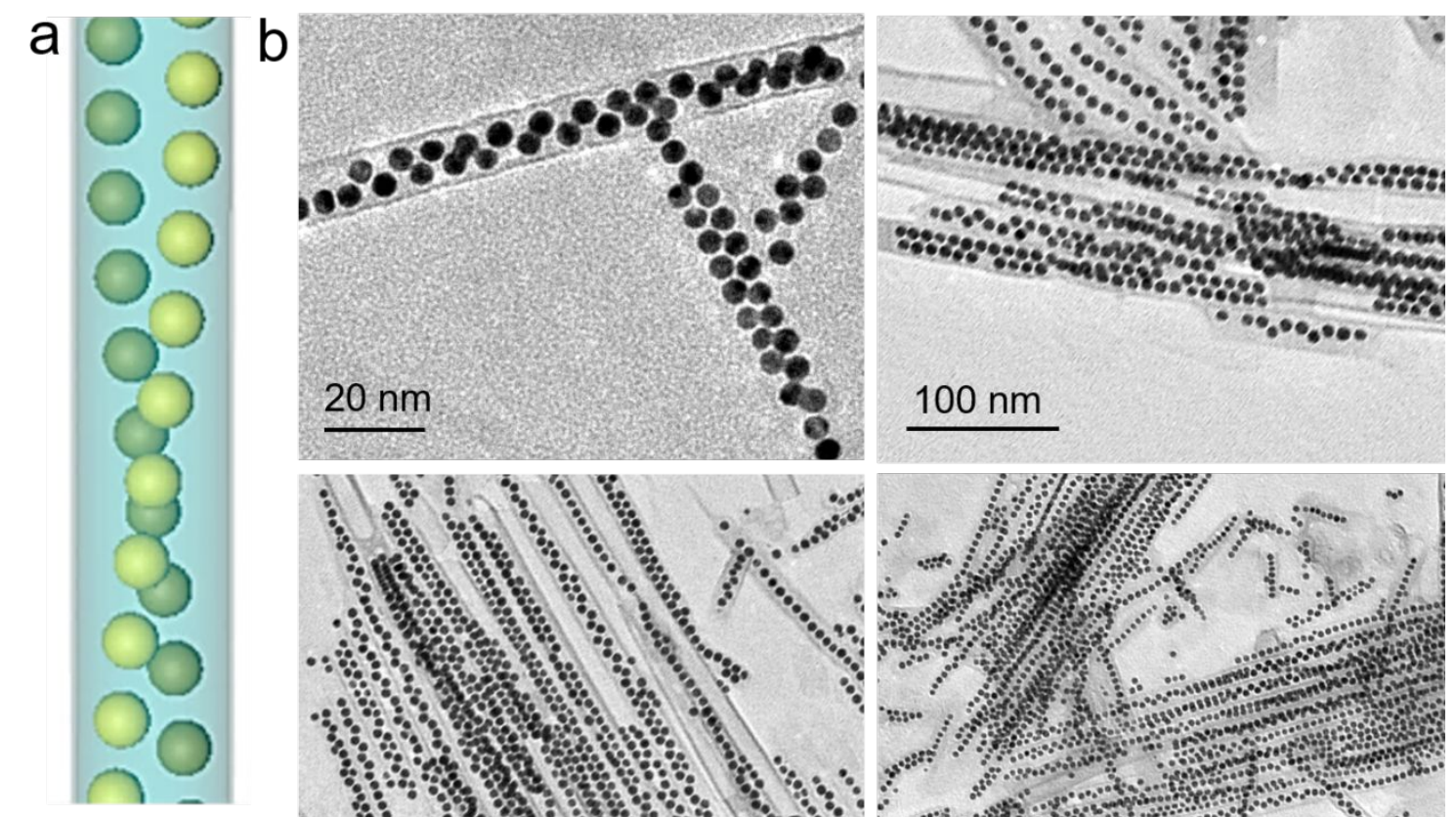

Double helix
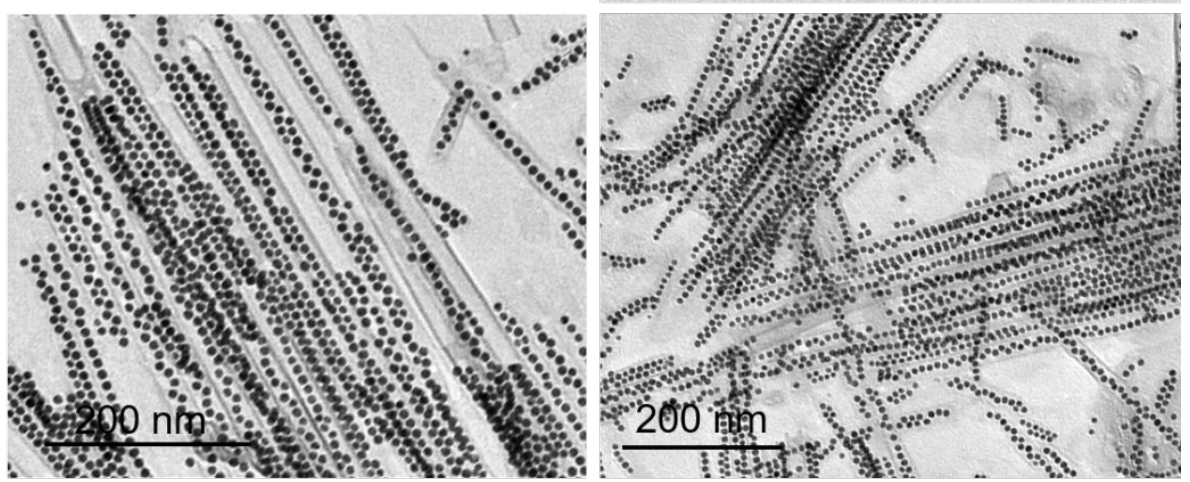

Figure S12. The model (a) and the corresponding TEM images (b) of Au6/NTs.

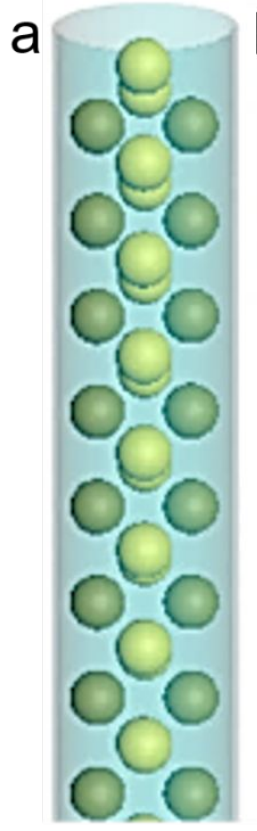

C2
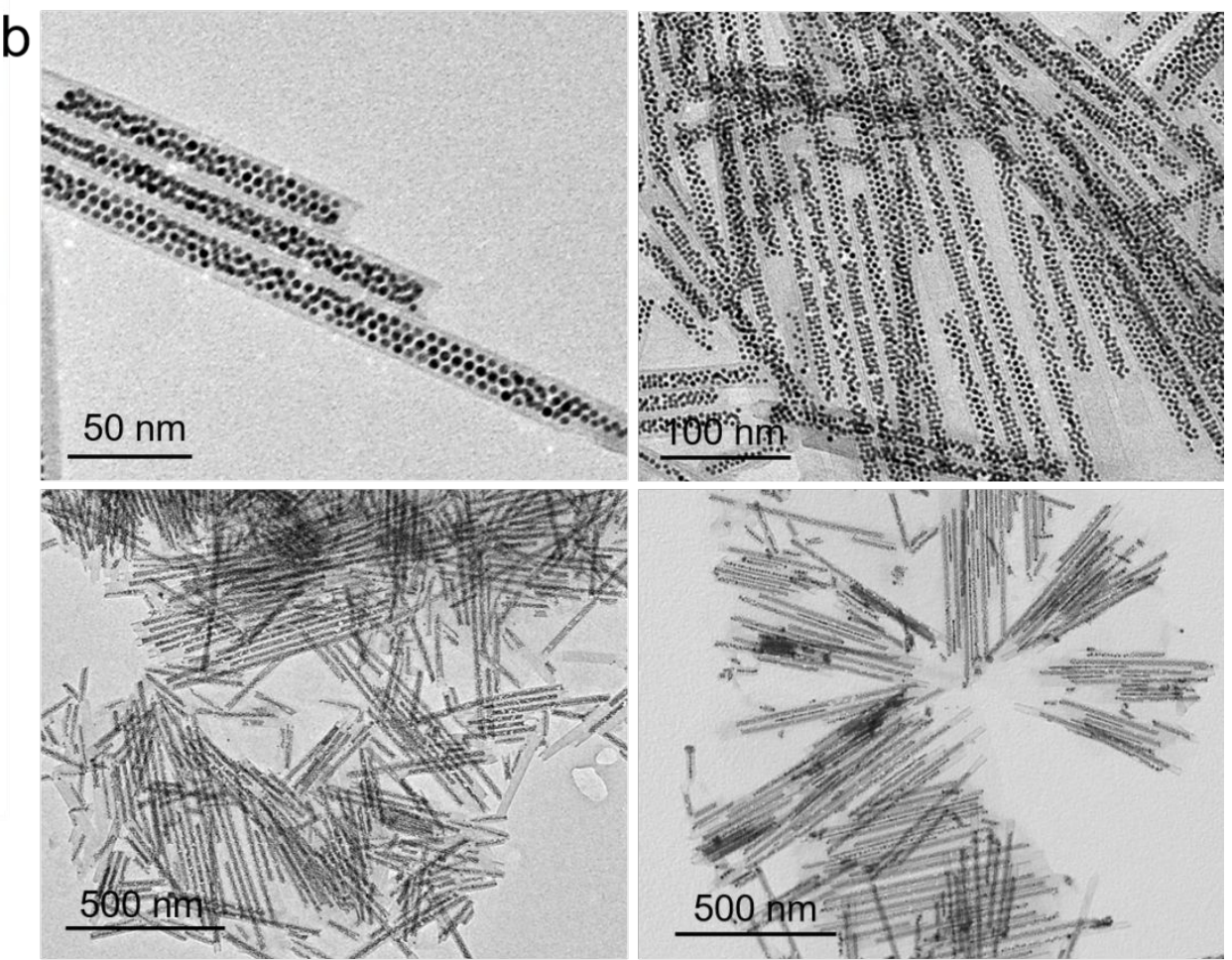

Figure S13. The model (a) and the corresponding TEM images (b) of Au5/NTs. 


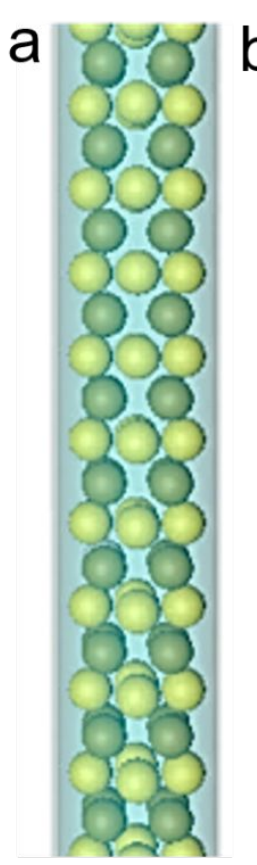

C4
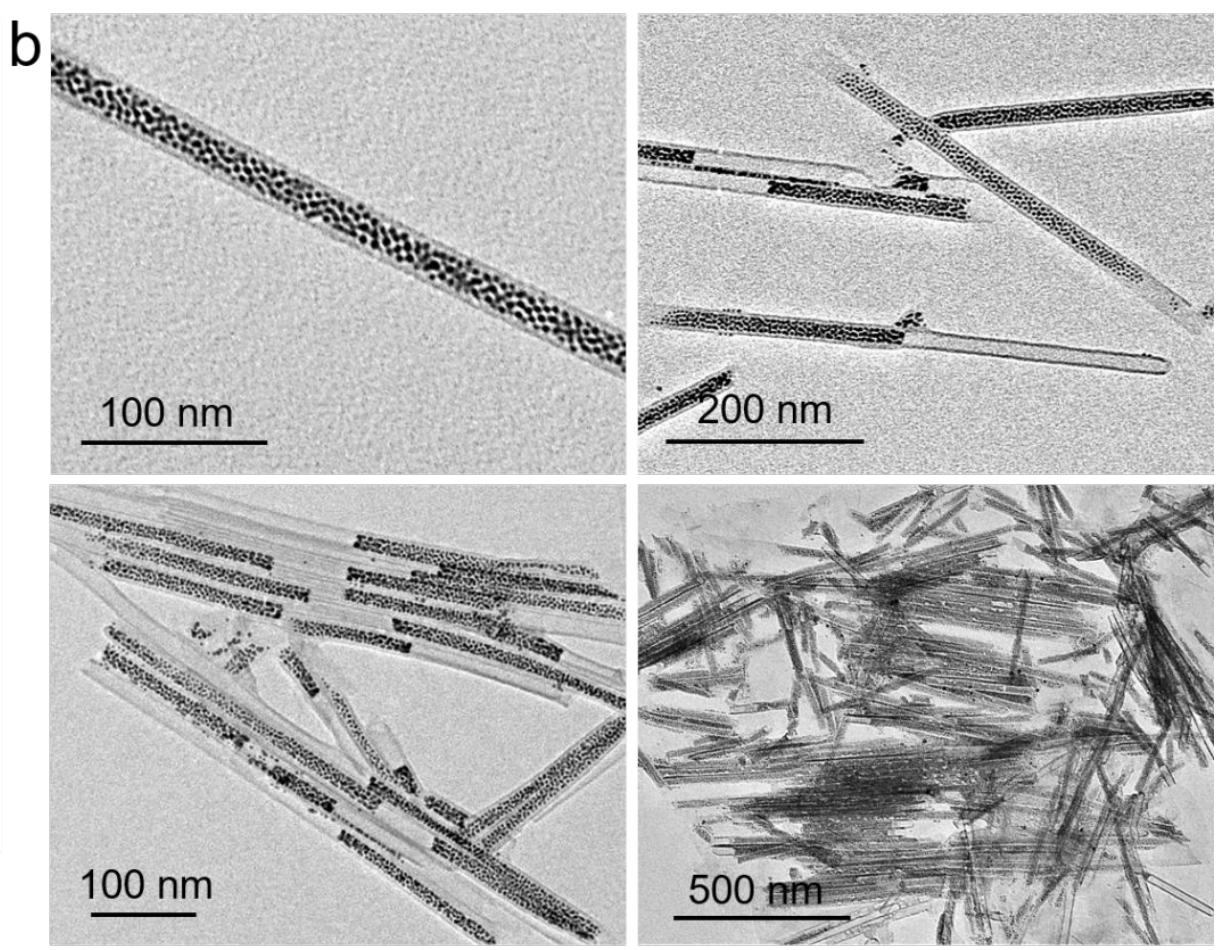

Figure S14. The model (a) and the corresponding TEM images (b) of Au4/NTs.
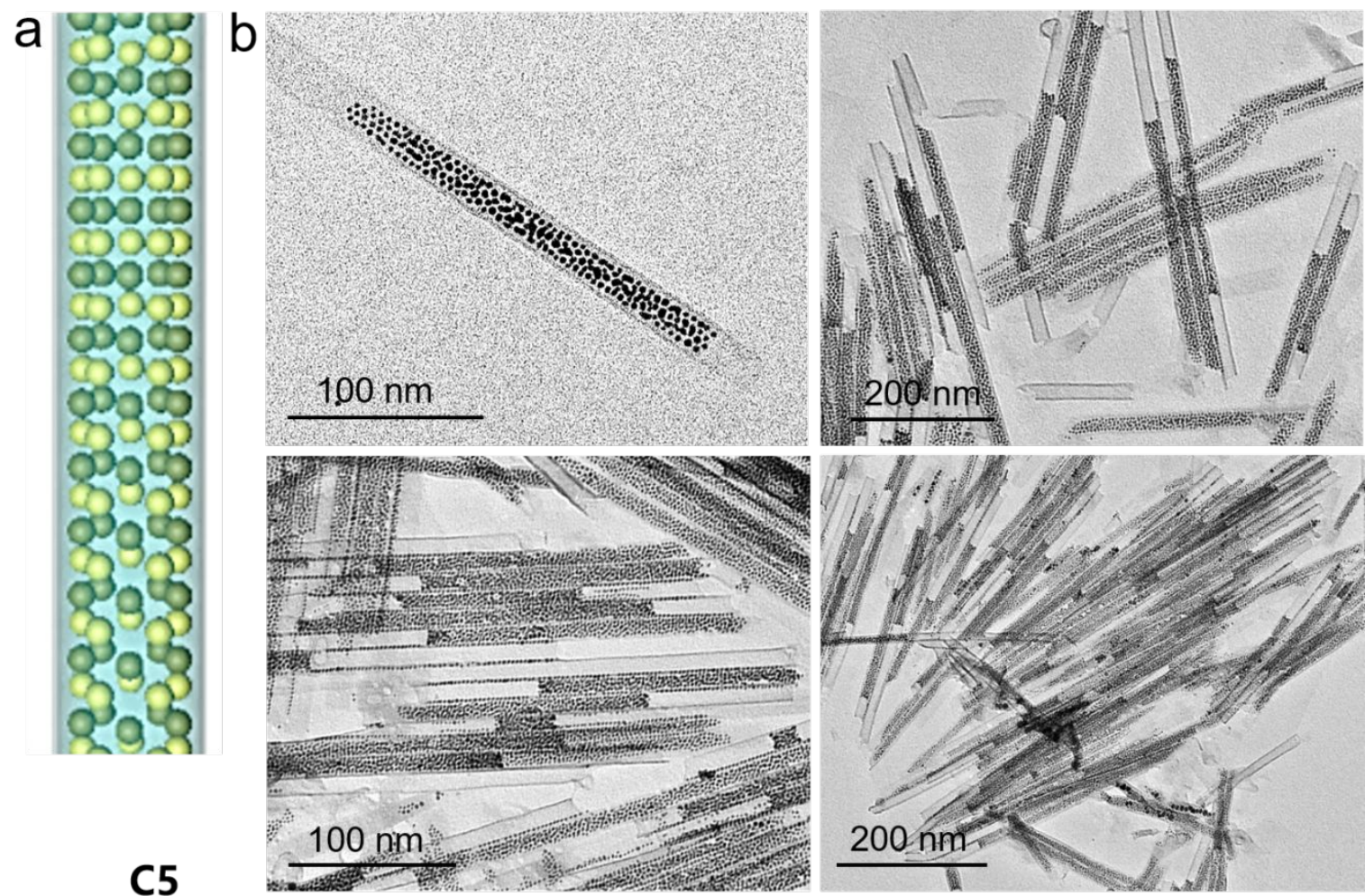

Figure S15. The model (a) and the corresponding TEM images (b) of Au3/NTs. 


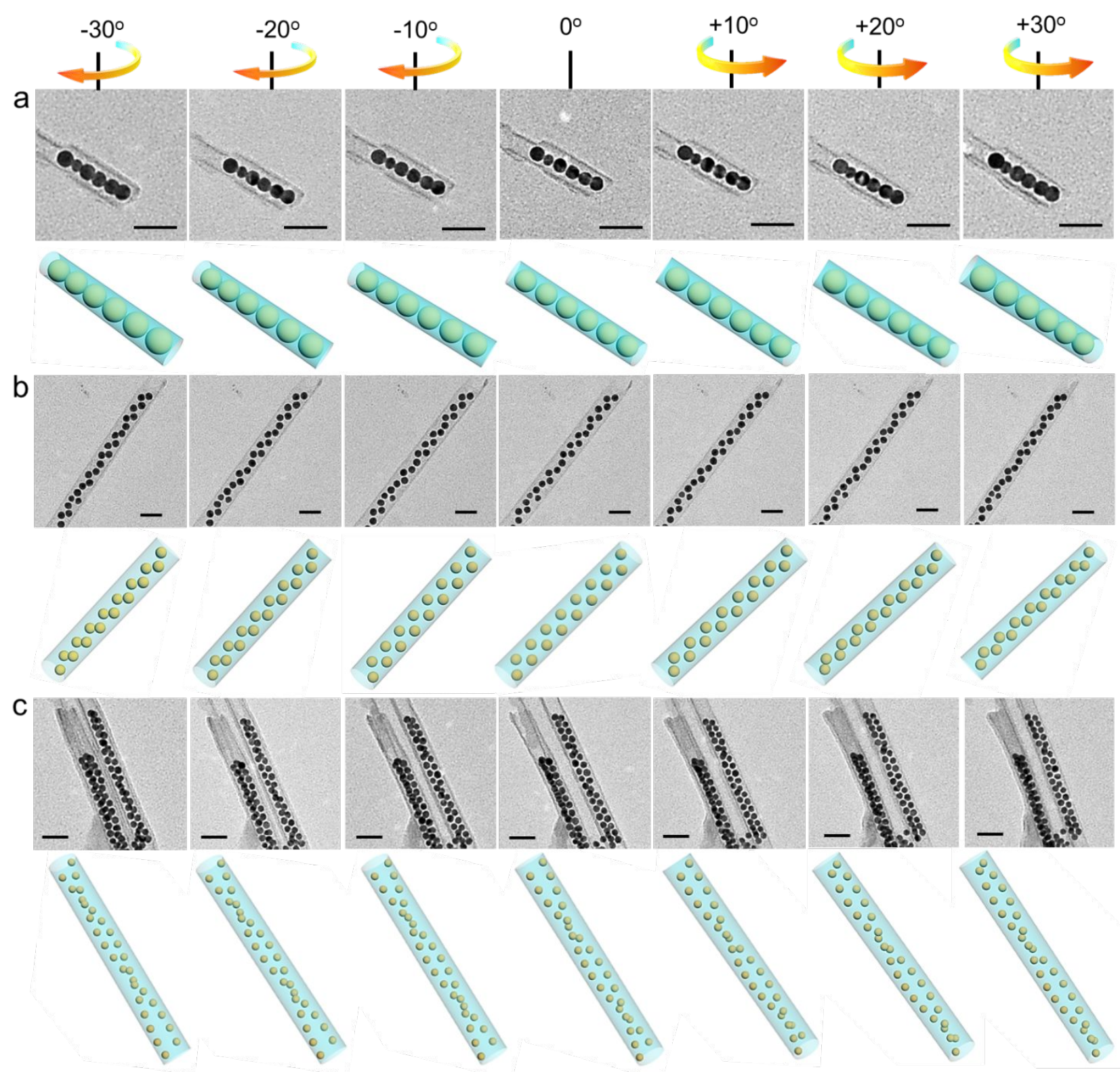

Figure S16. TEM tilting-angle experiments for (a) C1, (b) zigzag and (c) double helical superstructures. The tilting experiments were performed from $-30^{\circ}$ to $+30^{\circ}$. Furthermore, the tilting experiments were also compared with the models developed in 3DS Max software. For the $C 1$ symmetric structure (Figure S16a), the tilting angles from $-30^{\circ}$ to $+30^{\circ}$ results in the overlay between two adjacent NPs, which confirms the single NP chain-like structure. For the Zigzag structure (Figure S16b), from all the different tilting angles, the zigzag structure can be determined, which is in agreement with the structural model. For the double helix structure (Figure S16c), the different tilting angles can result in the different observations.

Additional comments on Figure S10-S16. The structural defects in the assembled structure can be 
due to the size dispersion of the NPs, and the size of NPs in the same sample varies from one to several nanometers. The slight difference in NP size can result in the stacking defects in the assembly.
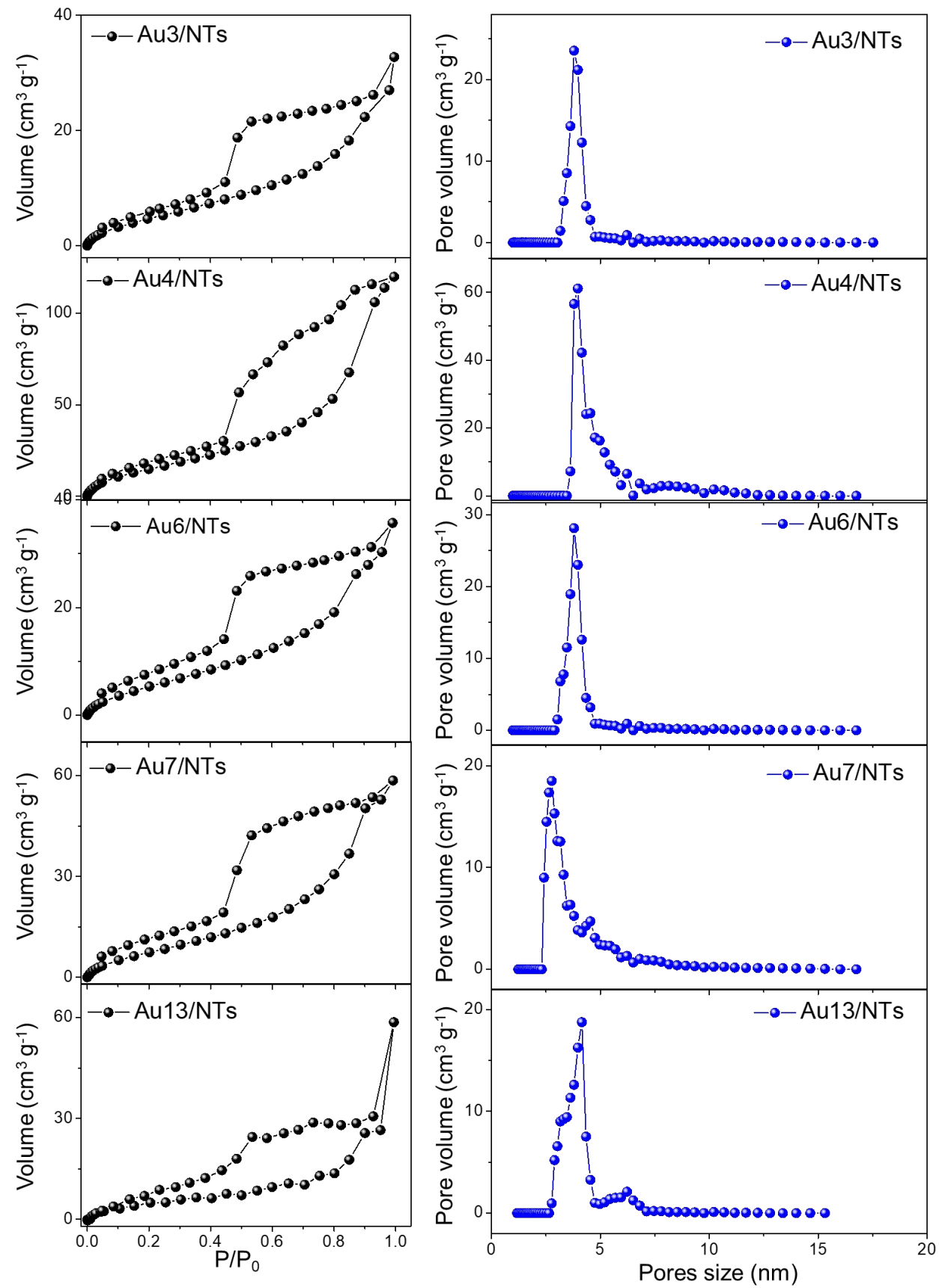

Figure S17. $\mathrm{N}_{2}$ adsorption-desorption isotherms and pore size distributions of Au3/NTs, Au4/NTs, Au6/NTs, Au7/NTs and Au13/NTs nanocomposites. The detailed BET surface areas and mean pore size can be found in Table S4. 
Table S4. The detailed BET surface areas and mean pore size of Au/NTs and Pd/NTs nanocomposites.

\begin{tabular}{ccc}
\hline Samples & Surface areas & Pore size \\
\hline NTs & 142 & 16.2 \\
Au3/NTs & 40 & 3.9 \\
Au4/NTs & 86 & 4.2 \\
Au5/NTs & 58 & 4.0 \\
Au6/NTs & 57 & 4.1 \\
Au7/NTs & 31 & 3.8 \\
Au13/NTs & 37 & 4.2 \\
Au3@NTs & 45 & 14.2 \\
Pd3/NTs & 118 & 4.5 \\
Pd3@NTs & 134 & 16.6 \\
\hline
\end{tabular}



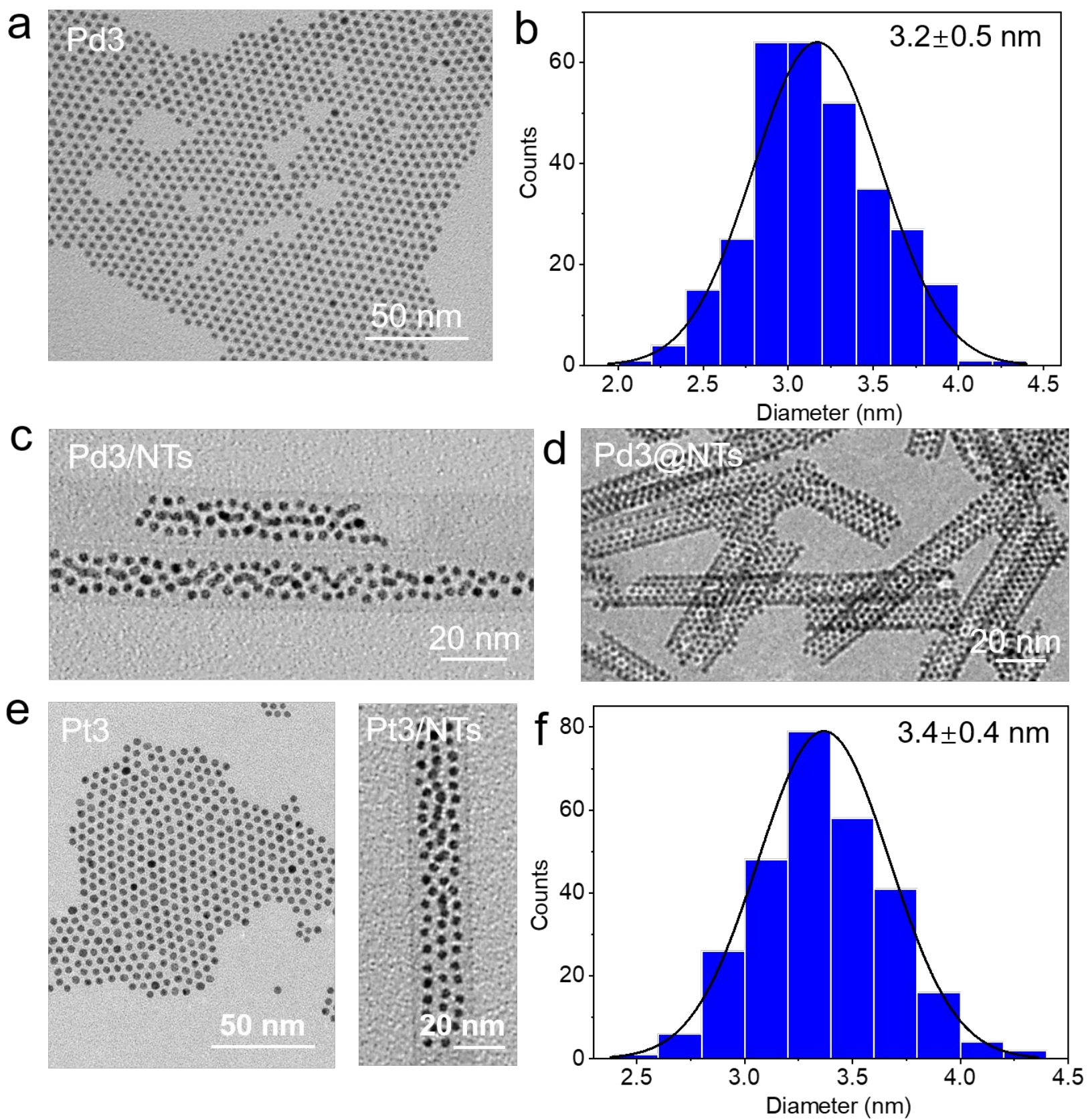

Figure S18. (a, b) Representative TEM images and size histograms of $3.2 \mathrm{~nm}$ Pd nanoparticles (Pd3), (c, d) Representative TEM images of Pd3/NTs and Pd3@NTs, (e) Representative TEM images of 3.4 nm Pt nanoparticles (Pt3) and Pd3/NTs and (f) size histograms of 3.4 nm Pt nanoparticles. 

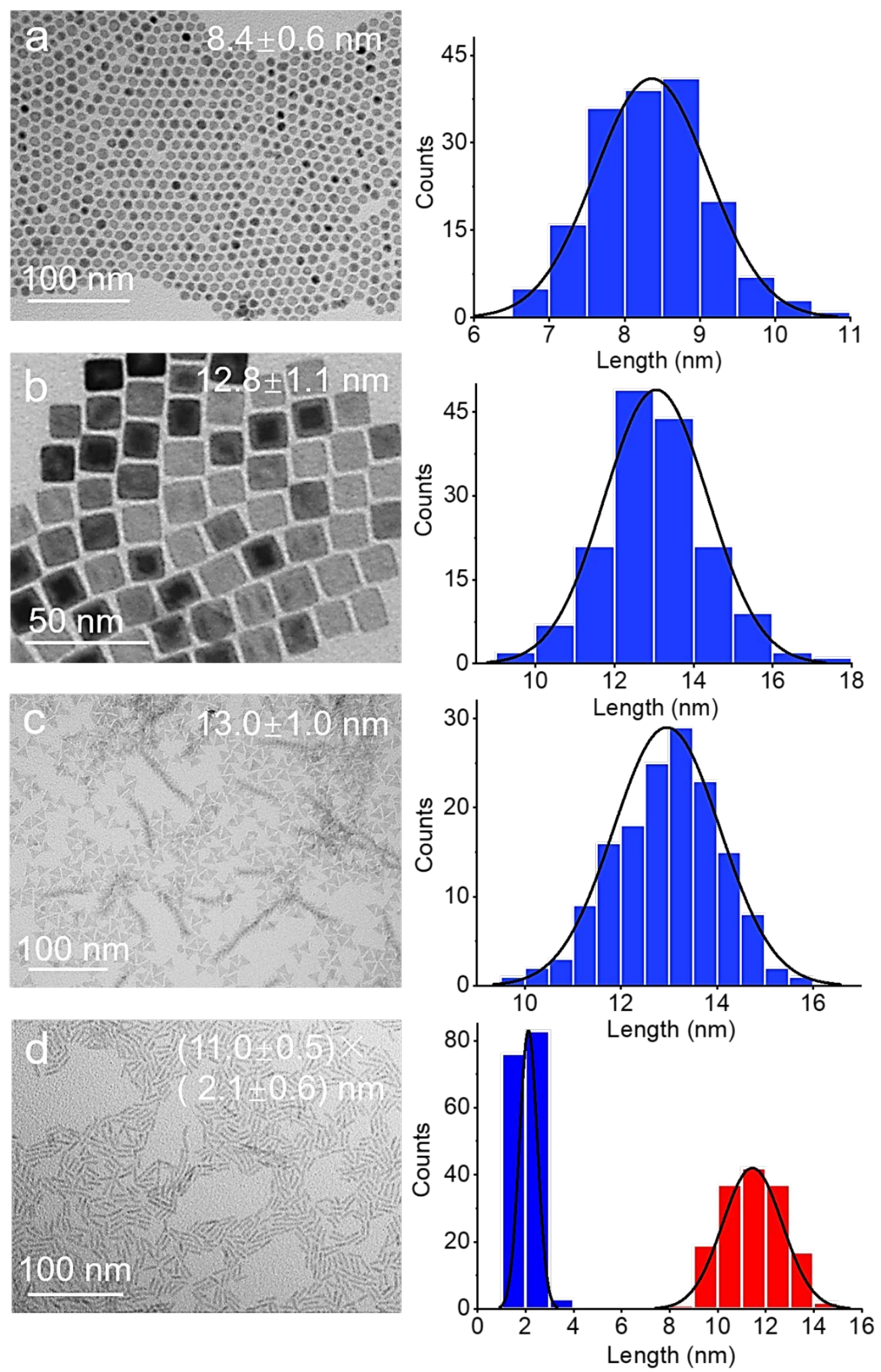

Figure S19. Representative TEM images of $\mathrm{Fe}_{3} \mathrm{O}_{4} \mathrm{NPs}(\mathbf{a}), \mathrm{Fe}_{3} \mathrm{O}_{4}$ nanocubes $(\mathbf{b}), \mathrm{Gd}_{2} \mathrm{O}_{3}$ nanoplatelets (c) and CdSe nanorods (d). The right part is the corresponding size histograms measured from 500 NPs. 
a

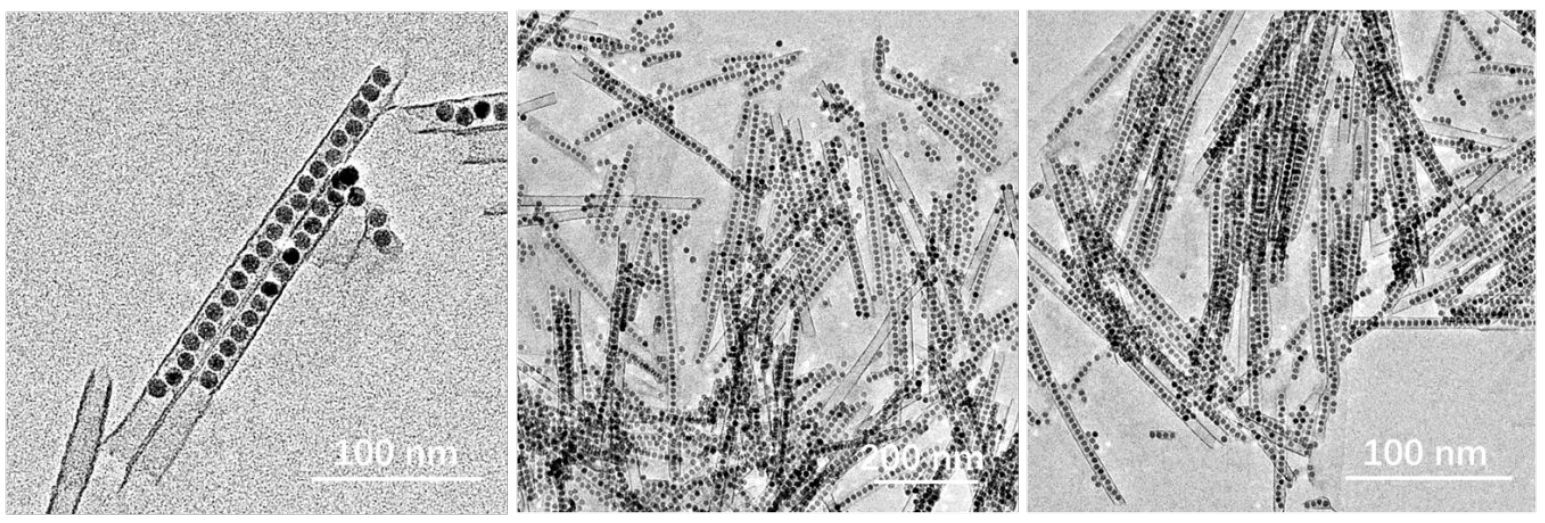

b
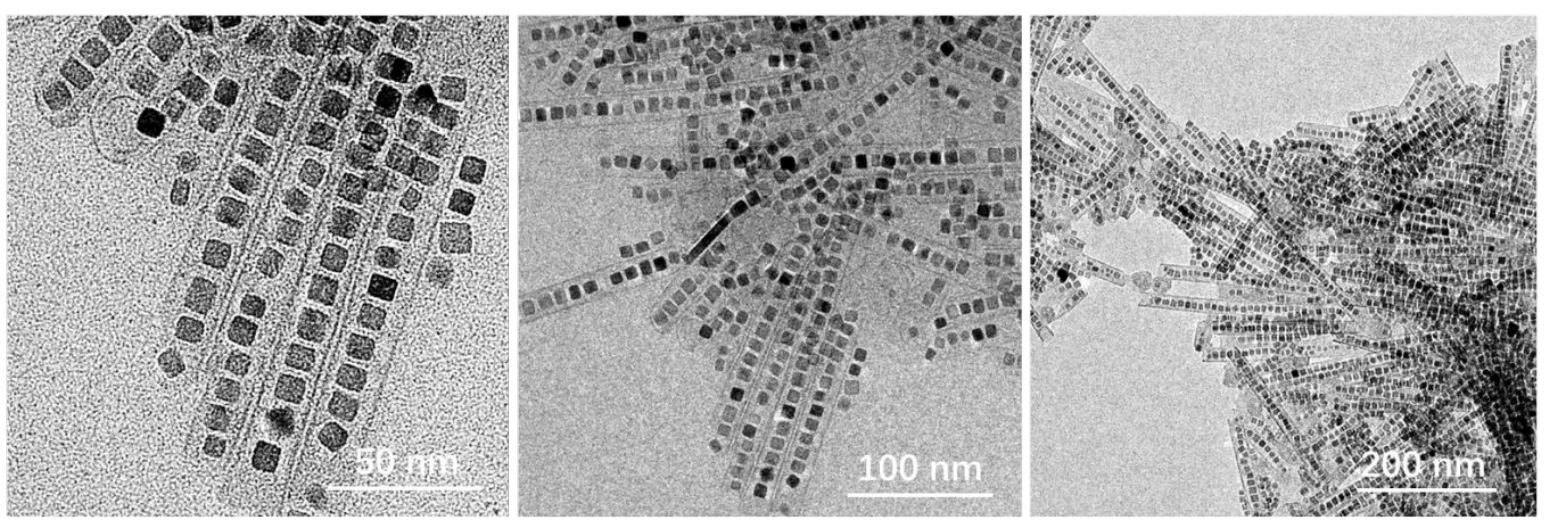

C
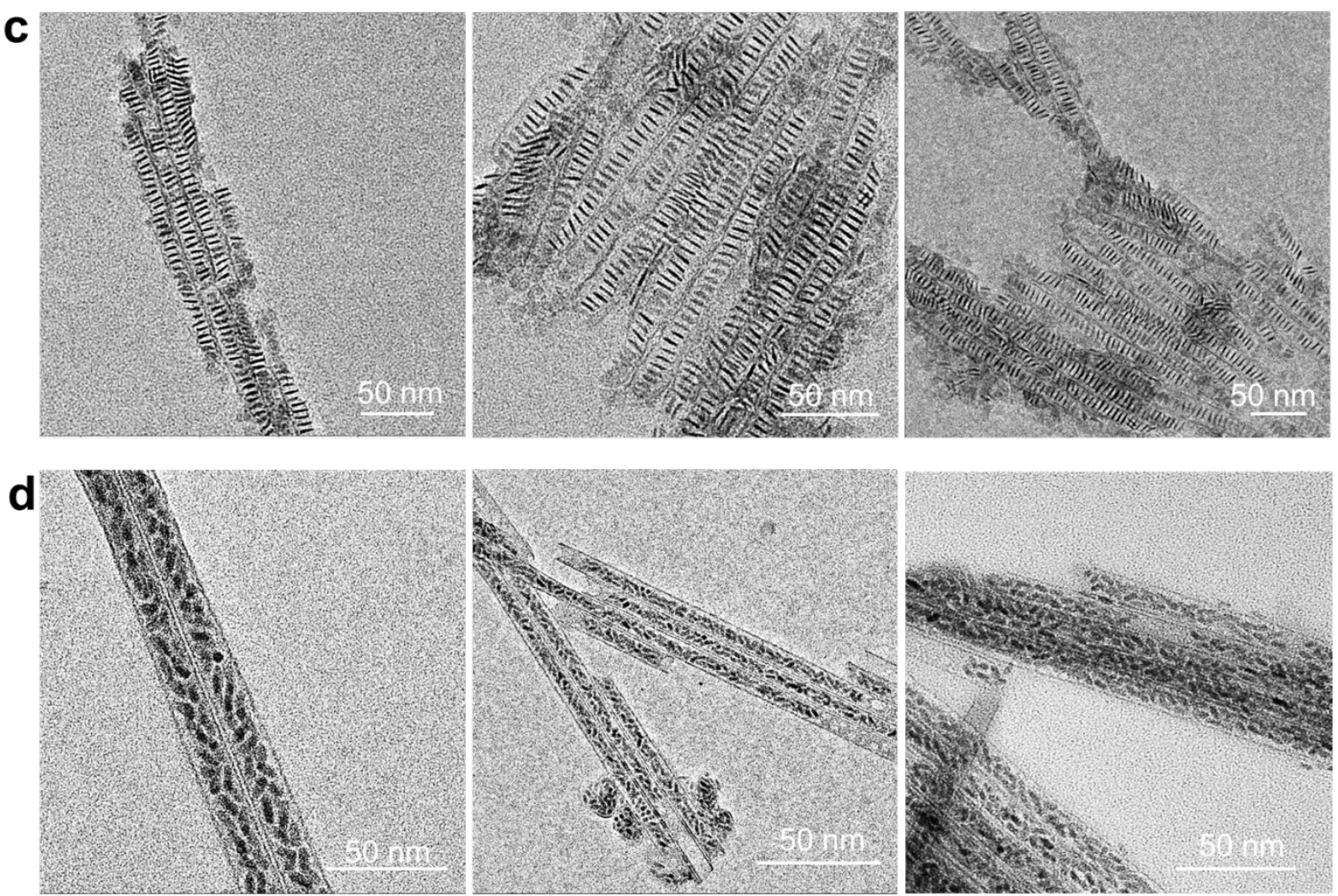

Figure S20. Additional TEM images of assemblies of $\mathrm{Fe}_{3} \mathrm{O}_{4} / \mathrm{NTs}(\mathbf{a}), \mathrm{Fe}_{3} \mathrm{O}_{4}$ nanocubes/NTs (b), $\mathrm{Gd}_{2} \mathrm{O}_{3} / \mathrm{NTs}(\mathbf{c})$ and CdSe nanorods/NTs (d). 

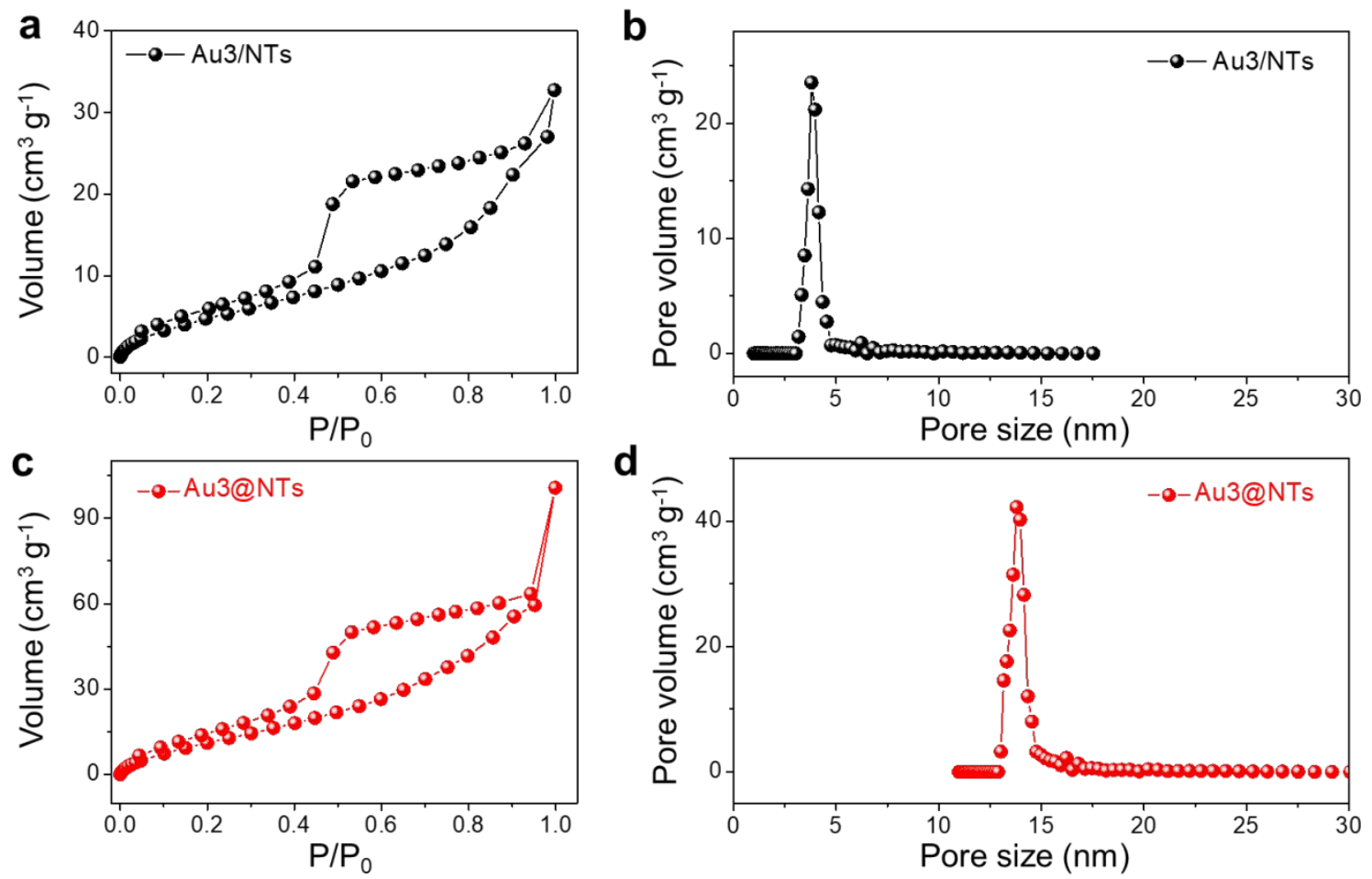

Figure S21. $\mathrm{N}_{2}$ adsorption-desorption isotherms and pore size distributions of Au3/NTs (a) and the corresponding pore size distributions (b). $\mathrm{N}_{2}$ adsorption-desorption isotherms and pore size distributions of Au3@NTs (c) and the corresponding pore size distributions (d).
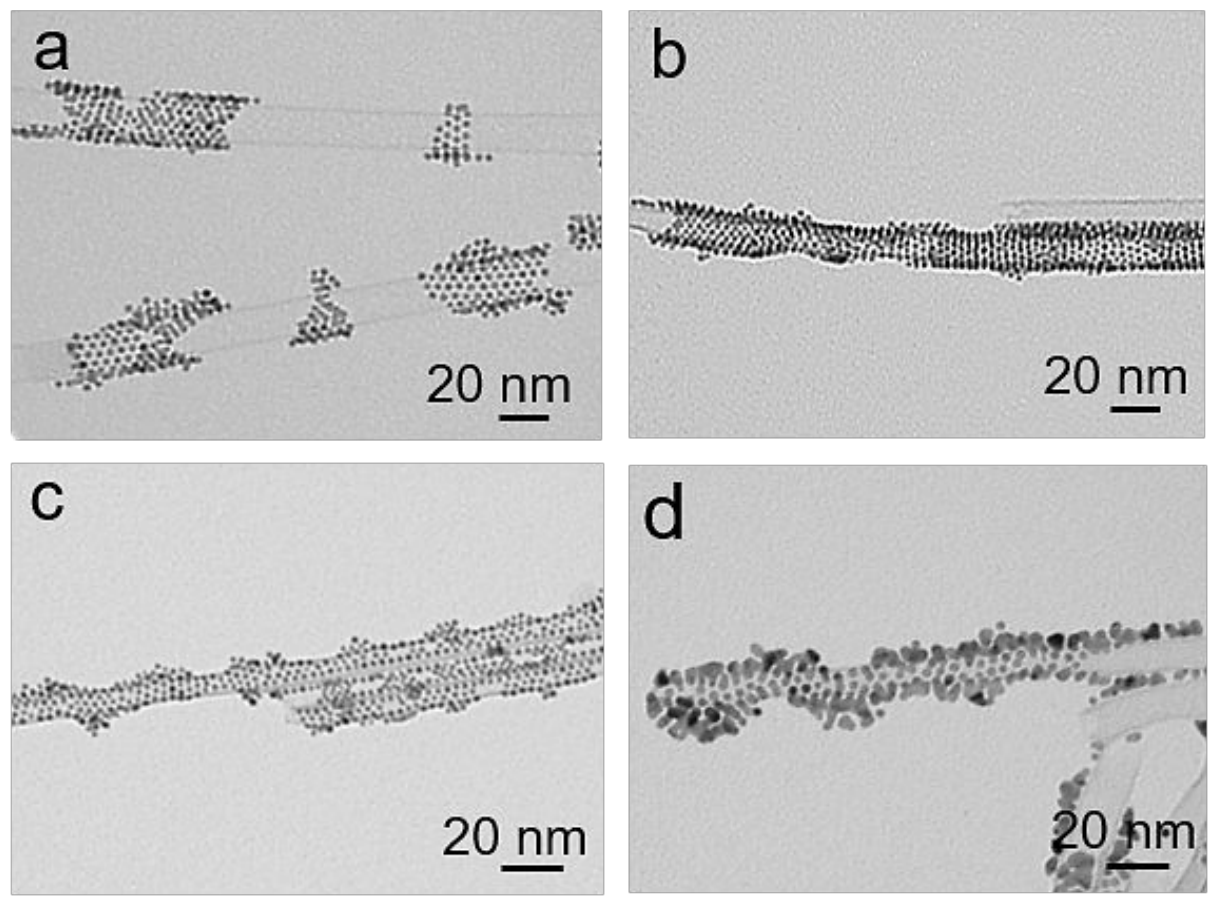
Figure S22. TEM images of Au3 NPs assembly outside the NTs with different amounts of limonene, $0.05 \mathrm{mmol}$ (a), $0.1 \mathrm{mmol}$ (b), $0.15 \mathrm{mmol}(\mathbf{c}), 0.2 \mathrm{mmol}(\mathbf{d})$.
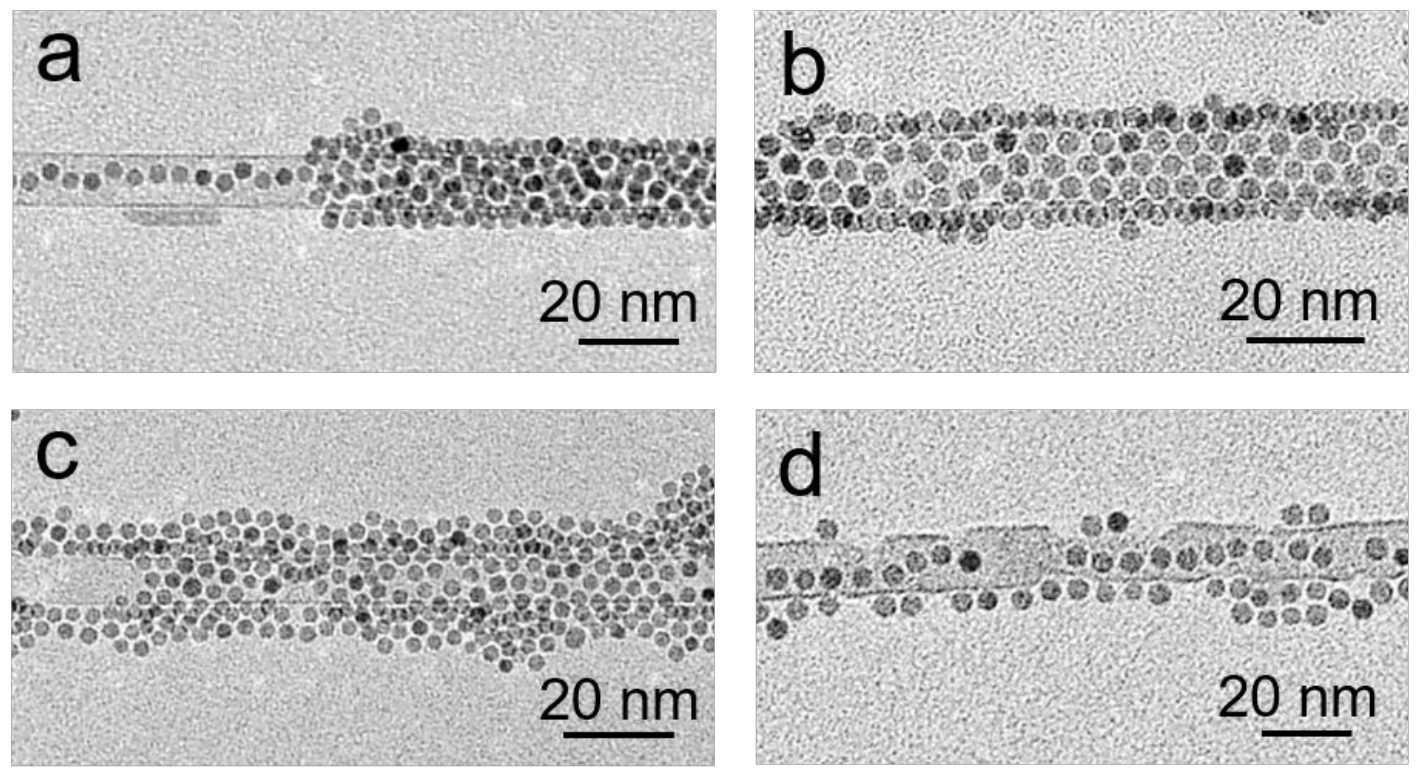

Figure S23. TEM images of $\mathrm{Fe}_{3} \mathrm{O}_{4}$ NPs assembly outside the NTs with different amounts of limonene, $0.05 \mathrm{mmol}$ (a), $0.1 \mathrm{mmol}$ (b), $0.15 \mathrm{mmol}$ (c), $0.2 \mathrm{mmol}$ (d). 

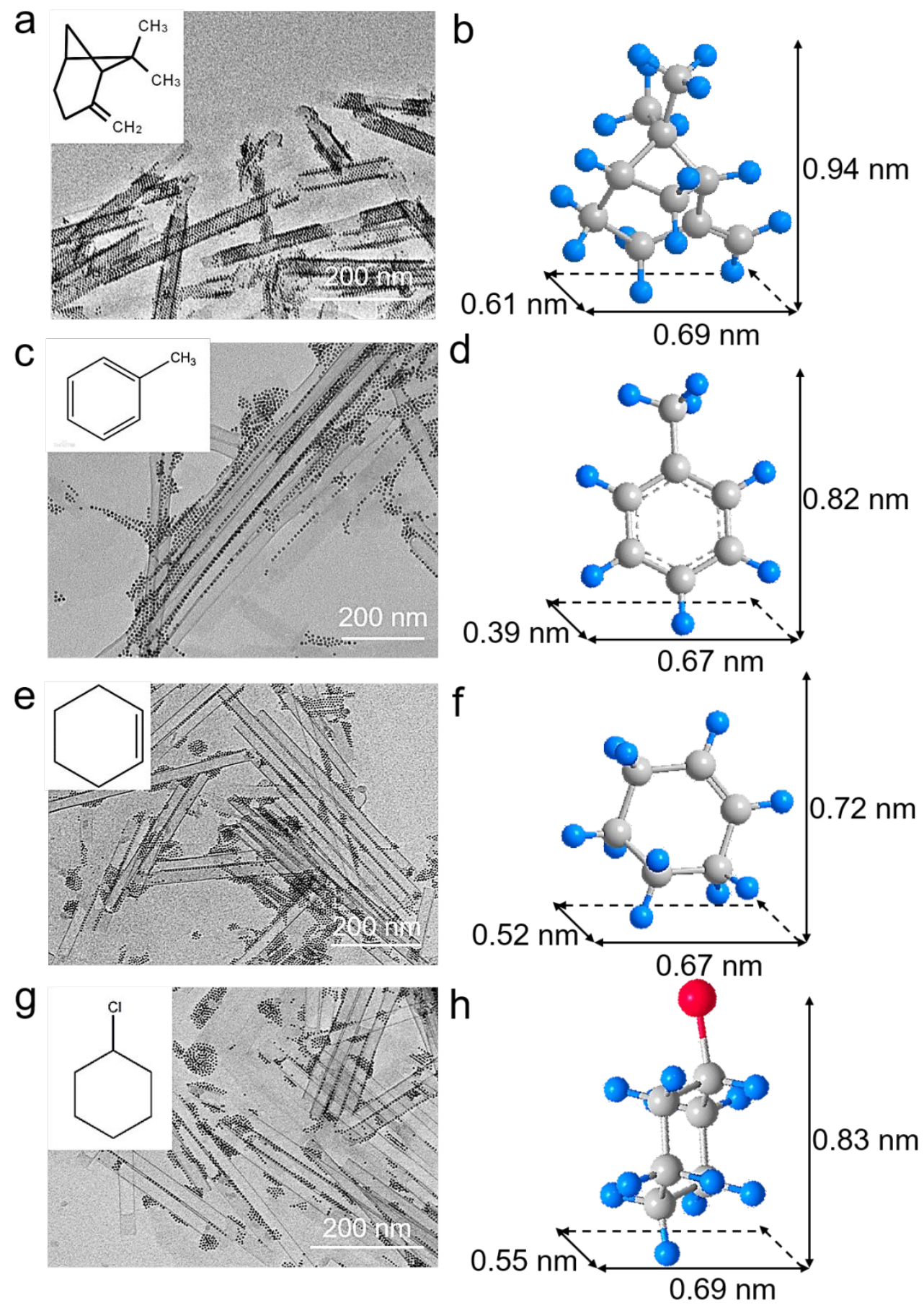

Figure S24. TEM images of small sized additives, such as $\beta$-pinene (a) and $\beta$-pinene with molecular size of $0.94 \times 0.69 \times 0.61 \mathrm{~nm}^{3}(\mathbf{b})$, result in NPs self-assembled into hexagonal close-packed monolayer structures at the external surface of NTs. However, smaller sized additives, such as toluene with molecular size of $0.82 \times 0.67 \times 0.39 \mathrm{~nm}^{3}(\mathbf{c}, \mathbf{d})$, cyclohexene with molecular size of $0.72 \times 0.67 \times 0.52 \mathrm{~nm}^{3}$ (e, f) and chlorocyclohexane with molecular size of $0.83 \times 0.69 \times 0.55 \mathrm{~nm}^{3}(\mathbf{g}, \mathbf{h})$, result in phase separations rather than host-guest or competitive assembly. 

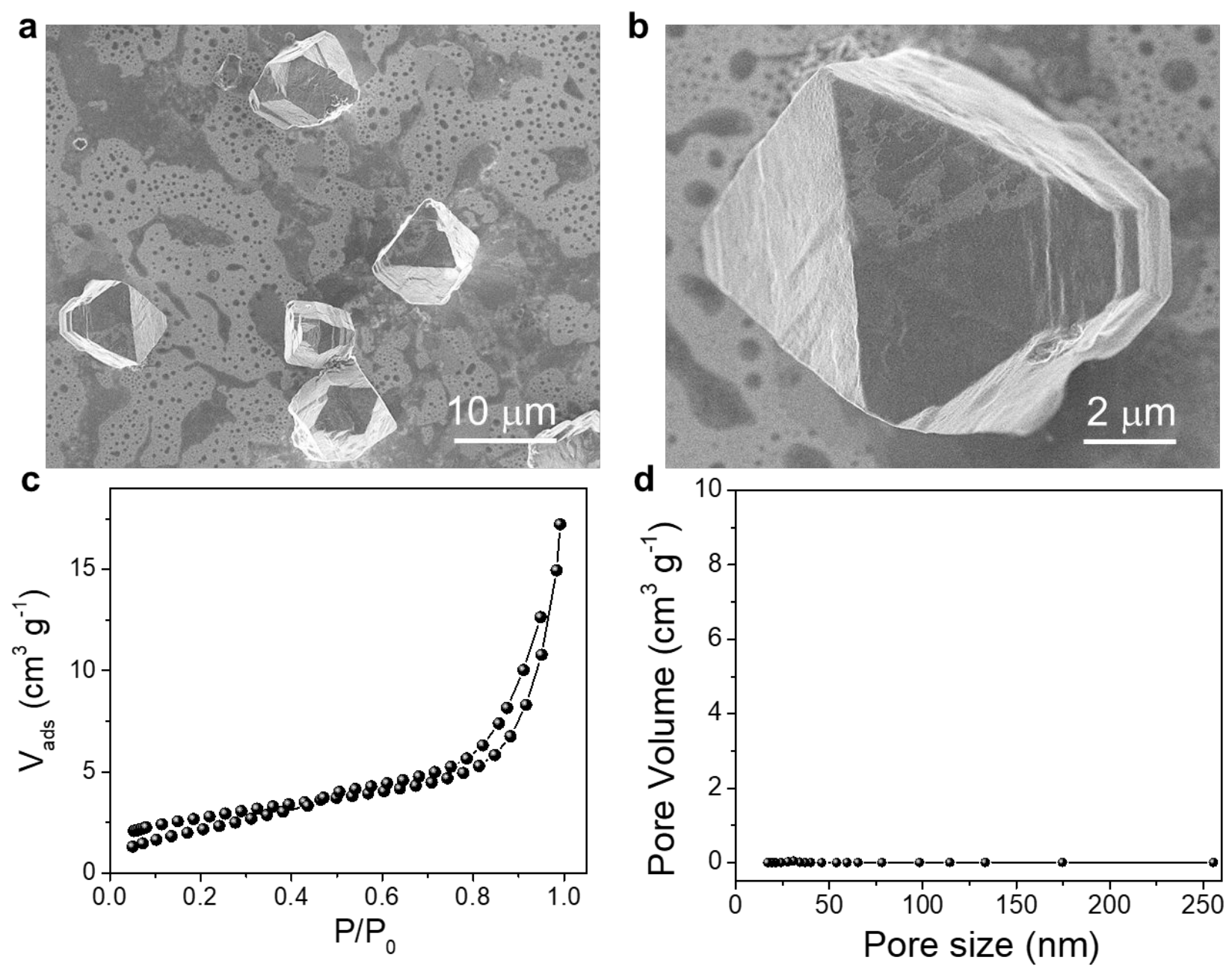

Figure S25. (a-b) SEM images of Au3 NP assemblies from the same conditions as described for Au3/NTs but in the absence of NTs; (c) Nitrogen adsorption-desorption isotherms of Au3 NP assemblies; (d) Pore size distribution of Au3 NP assemblies. 

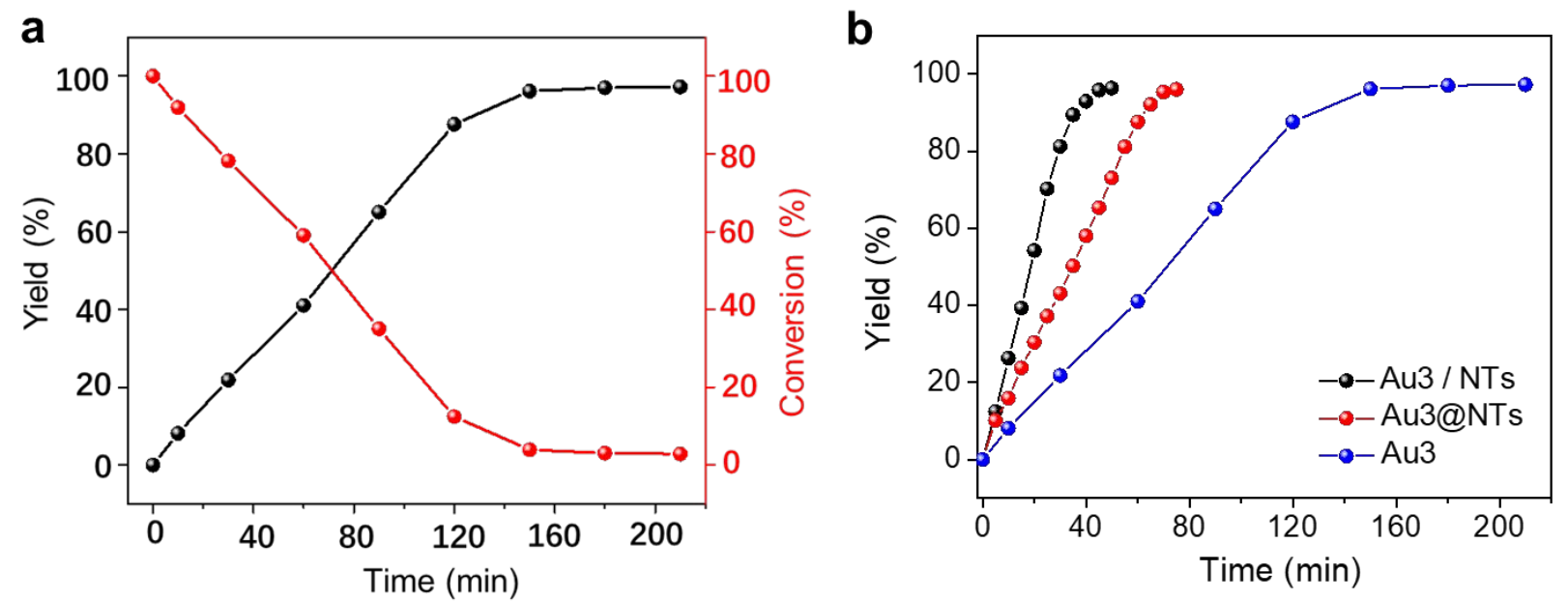

Figure S26. (a) time-dependent DPS alcoholysis over Au3 NP assemblies and (b) comparison of timedependent DPS alcoholysis over three different catalysts.

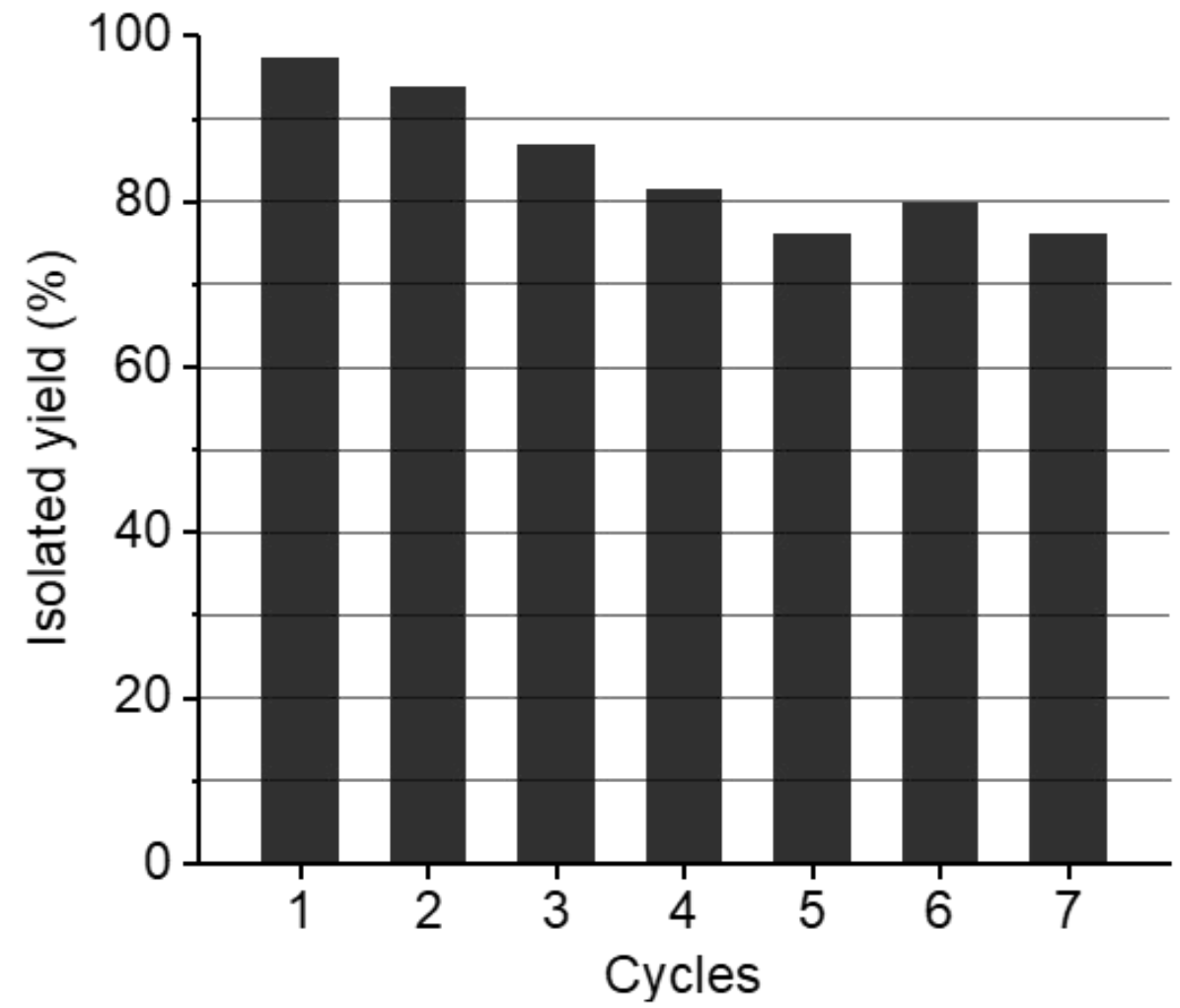

Figure S27. Stability studies: catalytic experiment using the recycled Au3/NTs catalyst for DPS methanolysis. 

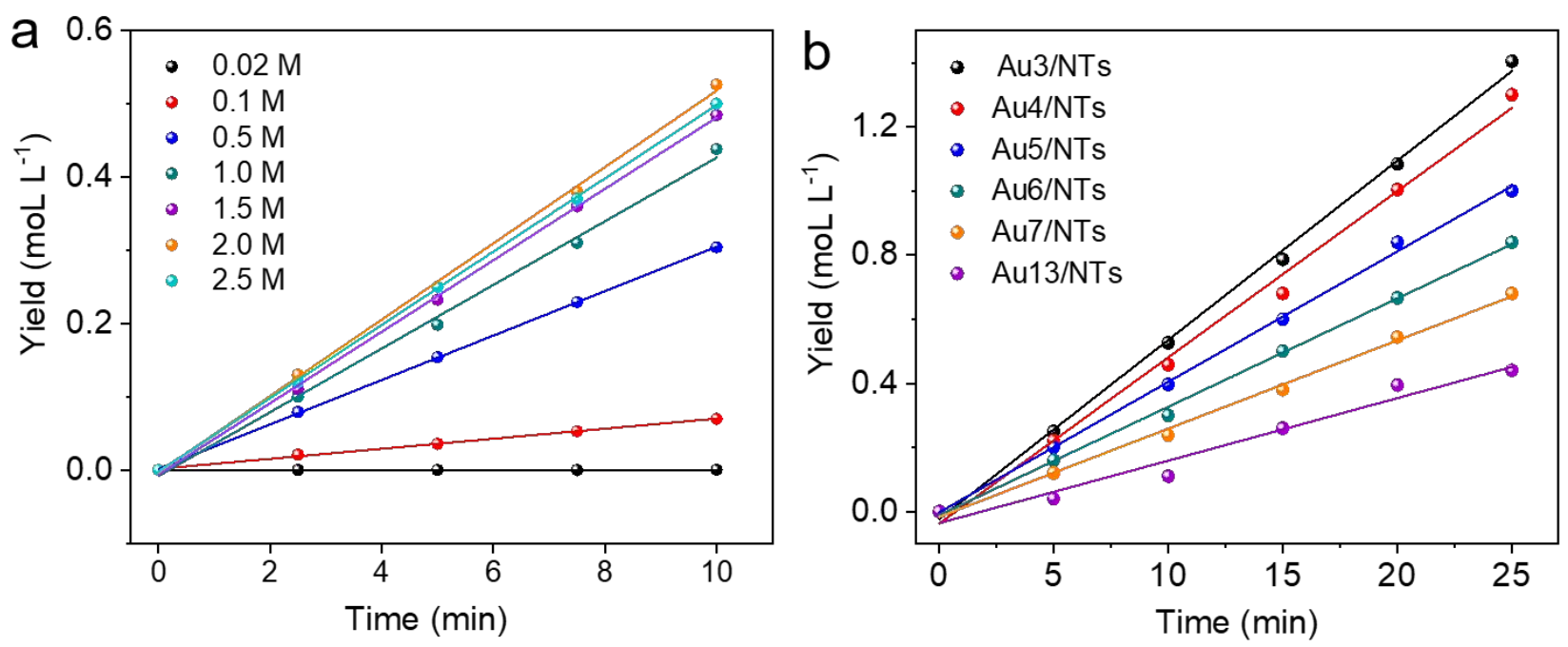

Figure S28. Plot of the amount of DPS against the alcoholysis activity (a) and the DPS concentration dependent catalytic alcoholysis activity. Plot of the different catalysts against the alcoholysis activity (b).
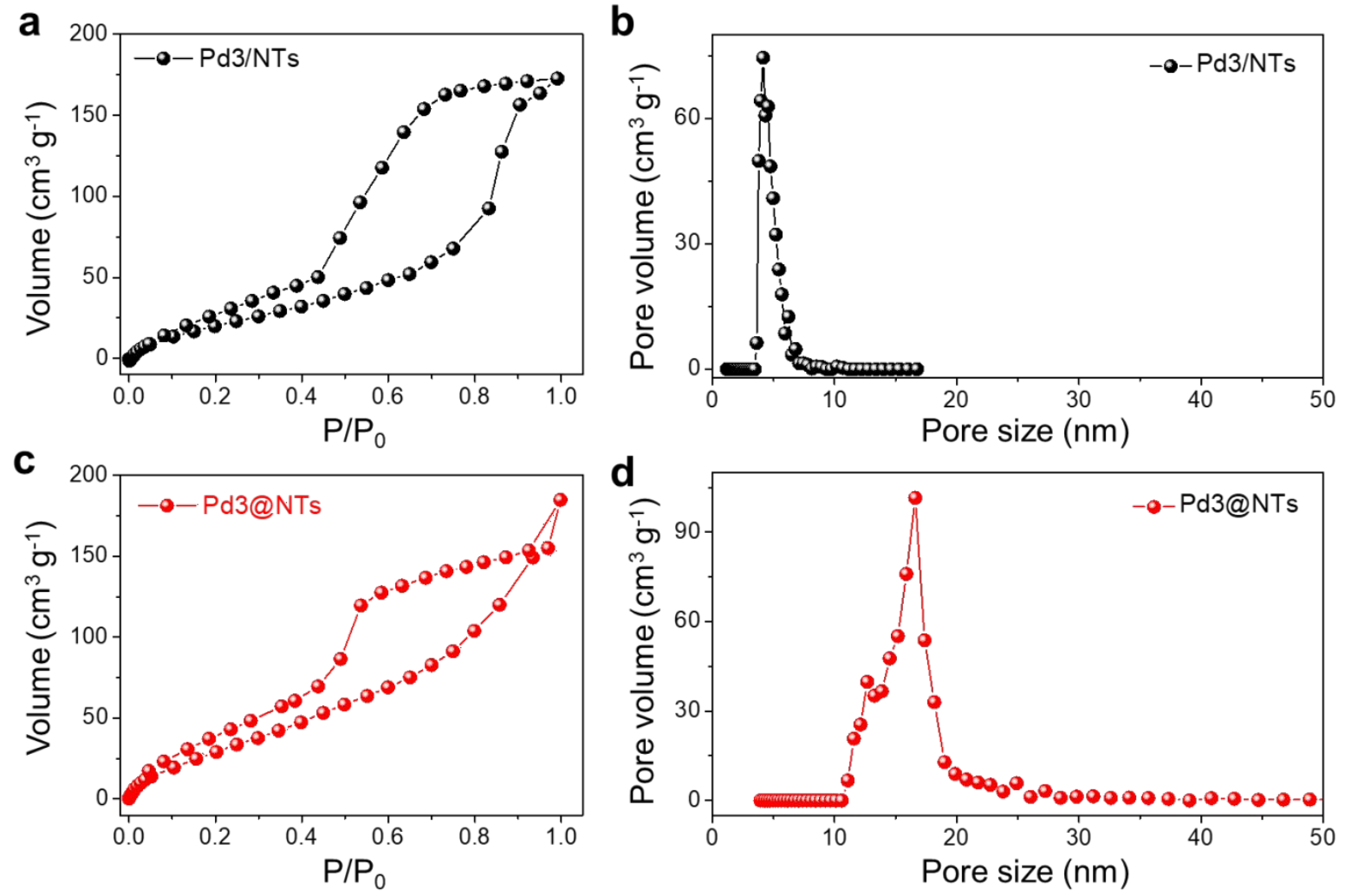

Figure S29. $\mathrm{N}_{2}$ adsorption-desorption isotherms and pore size distributions of $\mathrm{Pd} 3 / \mathrm{NTs}$ (a) and the corresponding pore size distributions (b). $\mathrm{N}_{2}$ adsorption-desorption isotherms and pore size distributions of Pd3@NTs (c) and the corresponding pore size distributions (d). The detailed BET 
surface areas and mean pore size can be found in Table S4.
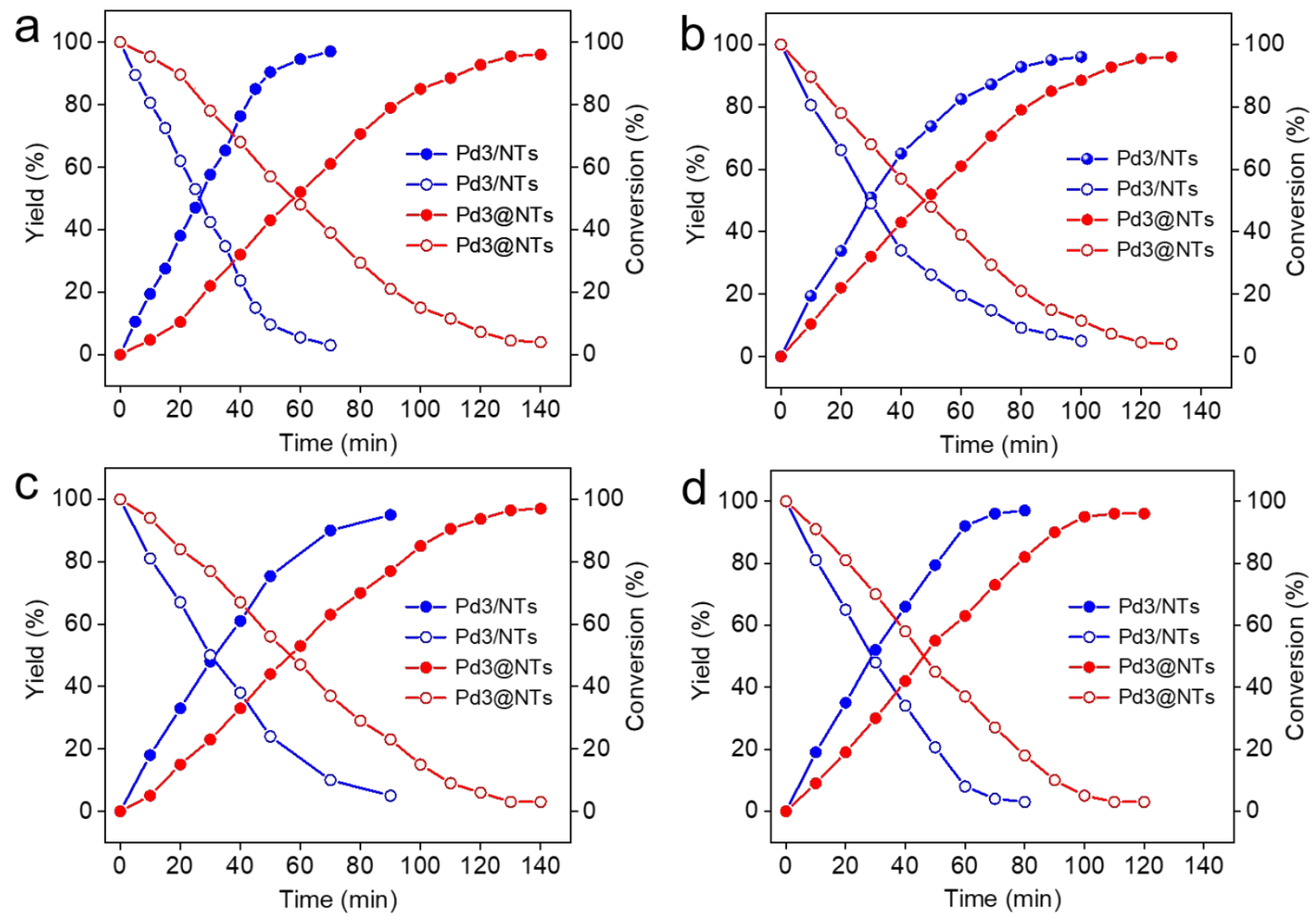

Figure S30. Time-dependent hydrogenation of alkynes to alkenes over Pd3/NTs and Pd3@NTs catalysts. (a-d) The corresponding alkynes are 1a (a), 1a (b), 1a (c) and 1a (d) (see Table 2). 


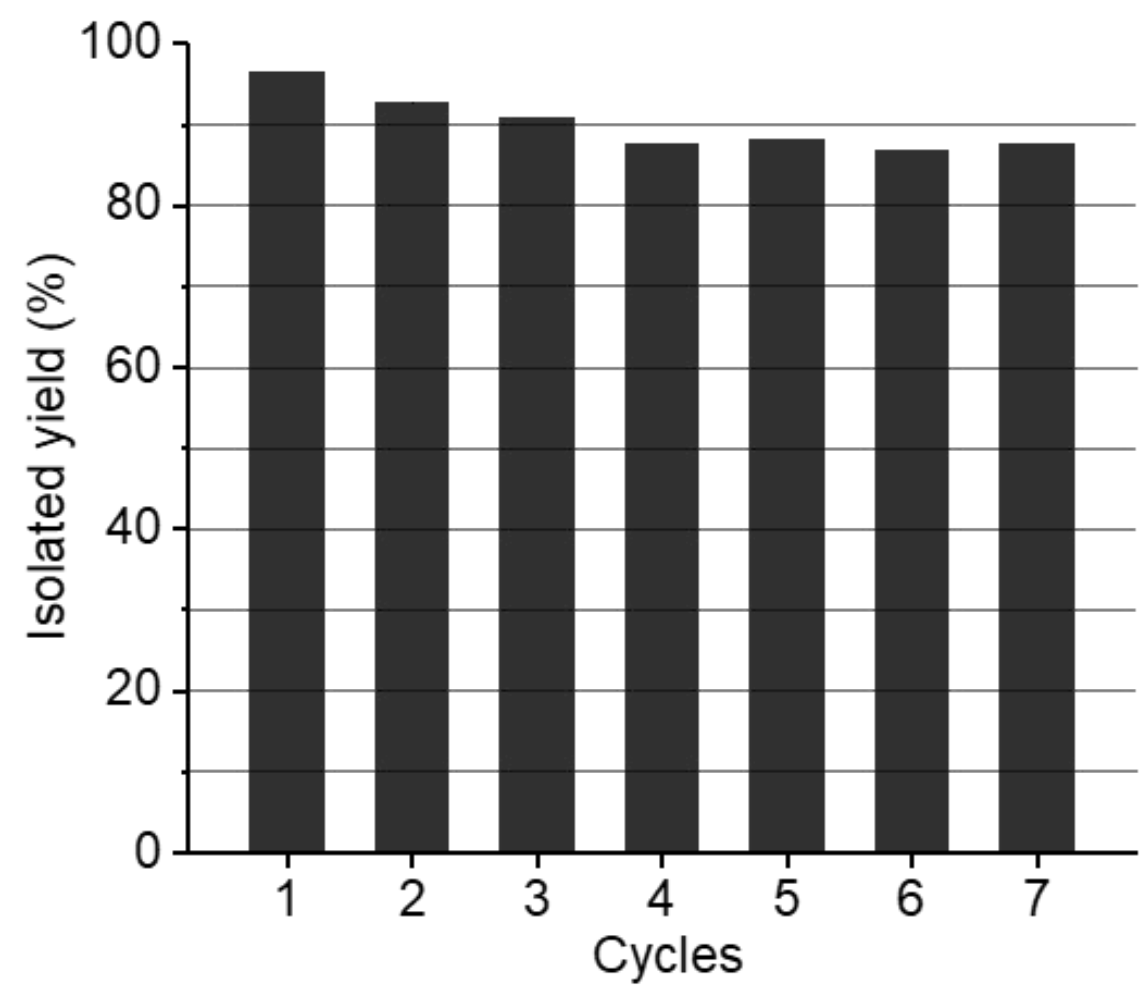

Figure S31. Stability studies: catalytic experiment using the recycled Pd3/NTs catalyst for selective hydrogenation of dimethyl acetylene dicarboxylate to alkenes. 


\section{Movie captions.}

Movie S1. Atomistic simulations of trapping of DPS molecules into $C 4$ symmetric porous structures (Color Code: Gold in yellow, DPS in red and dodecanethiol in purple, methanol molecules omitted for clarity). 